\title{
High Order Space-Time Adaptive WENO Finite Volume Schemes for Non-Conservative Hyperbolic Systems
}

\author{
Michael Dumbser ${ }^{\mathrm{a}, 1}$, Arturo Hidalgo ${ }^{\mathrm{b}}$, Olindo Zanotti ${ }^{\mathrm{a}}$ \\ ${ }^{a}$ Laboratory of Applied Mathematics, Department of Civil, Environmental and \\ Mechanical Engineering, University of Trento, I-38123 Trento, Italy \\ ${ }^{b}$ Departamento de Matemática Aplicada y Métodos Informáticos, Universidad Politécnica \\ de Madrid, 28003-Madrid, Spain
}

\begin{abstract}
We present a class of high order finite volume schemes for the solution of nonconservative hyperbolic systems that combines the one-step ADER-WENO finite volume approach with space-time adaptive mesh refinement (AMR). The resulting algorithm, which is particularly well suited for the treatment of material interfaces in compressible multi-phase flows, is based on: (i) high order of accuracy in space obtained through WENO reconstruction, (ii) a high order one-step time discretization via a local space-time discontinuous Galerkin predictor method, and (iii) the use of a path conservative scheme for handling the non-conservative terms of the equations. The AMR property with time accurate local time stepping, which has been treated according to a cell-by-cell strategy, strongly relies on the high order one-step time discretization, which naturally allows a high order accurate and consistent computation of the jump terms at interfaces between elements using different time steps. The new scheme has been succesfully validated on some test problems for the Baer-Nunziato model of compressible multiphase flows.
\end{abstract}

Keywords: Adaptive Mesh Refinement (AMR), time accurate local time stepping, high order ADER approach, path-conservative WENO finite volume schemes, compressible multi-phase flows, Baer-Nunziato model

\footnotetext{
${ }^{1}$ michael.dumbser@unitn.it (M. Dumbser)
} 


\section{Introduction}

Physical phenomena governed by non-conservative hyperbolic systems arise in many scientific and technological areas, such as aerospace and automotive industry, geophysical flows, compressible multi-phase flows, oil and gas extraction, among many others. The mathematical modelling of these phenomena is very complicated and so far no universally accepted model exists. A common problem in many mathematical models used in the fields listed above is that the governing PDE system can not be written in fully conservative form. A special problem that concerns compressible multi-phase flows is the accurate resolution of material interfaces over long times. Among possible strategies to achieve a good resolution of material interfaces are the use of Lagrangian methods [64, 19, 18, 20, 27, 61, 34], ghost-fluid and levelset methods [44, 45, 72, 67, 47], little dissipative Riemann solvers combined with high order schemes [86, 90, 40] and, last but not least, the use of adaptive mesh refinement (AMR). Therefore, in this paper we suggest to combine high order accurate WENO finite volume schemes with AMR for the solution of compressible multi-phase flow problems to assure an accurate resolution of the material interfaces.

One particular and rather widespread mathematical model used in multiphase flow applications is based on the deflagration-to-detonation transition (DDT) theory, from which the Baer-Nunziato equations [7] can be derived. Reduced models have been proposed in [55, 69, 93], where the original BaerNunziato system has been simplified by carrying out an asymptotic analysis in the limit of stiff relaxation source terms.

Other models in the field of multi-phase flows are those proposed and analyzed by Saurel and Abgrall [78, 5, 79, 66] for the mixture of two compressible fluids, the depth-averaged debris-flow model by Pitman and Le [75] as well as single and multi-layer shallow water equations [24, 73, 3].

All the models above can be cast into the following general form of a nonlinear system of PDE in multiple space dimensions

$$
\frac{\partial \mathbf{u}}{\partial t}+\nabla \cdot \mathbf{F}(\mathbf{u})+\mathbf{B}(\mathbf{u}) \cdot \nabla \mathbf{u}=\mathbf{S}(\mathbf{u})
$$

where $\mathbf{u}$ is the state vector; $\mathbf{F}(\mathbf{u})=[\mathbf{f}(\mathbf{u}), \mathbf{g}(\mathbf{u}), \mathbf{h}(\mathbf{u})]$ is the flux tensor for the conservative part of the PDE system, with $\mathbf{f}(\mathbf{u}), \mathbf{g}(\mathbf{u})$ and $\mathbf{h}(\mathbf{u})$ expressing the fluxes along the $x, y$ and $z$ directions, respectively; $\mathbf{B}(\mathbf{u})=$ $\left[\mathbf{B}_{1}(\mathbf{u}), \mathbf{B}_{2}(\mathbf{u}), \mathbf{B}_{3}(\mathbf{u})\right]$ represents the non-conservative part of the system, 
written in block-matrix notation. Finally, $\mathbf{S}(\mathbf{u})$ is the source term, which may in principle be stiff. When written in quasilinear form, the system (1) becomes

$$
\frac{\partial \mathbf{u}}{\partial t}+\mathbf{A}(\mathbf{u}) \cdot \nabla \mathbf{u}=\mathbf{S}(\mathbf{u})
$$

where the matrix $\mathbf{A}(\mathbf{u})=\left[\mathbf{A}_{1}, \mathbf{A}_{2}, \mathbf{A}_{3}\right]=\partial \mathbf{F}(\mathbf{u}) / \partial \mathbf{u}+\mathbf{B}(\mathbf{u})$ contains both the conservative and the non-conservative contributions.

Recent work on numerical schemes for systems of equations involving non-conservative terms, like Eq. (1), includes the family of so-called pathconservative schemes [24, 49, 23, 74] which are based on the theory proposed by Dal Maso, Le Floch and Murat [65] and are a generalization of the usual concept of conservative methods for systems of conservation laws. Note that the weak formulation of the Roe method by Toumi [91] can also be considered as a path-conservative scheme. It has to be clearly stressed that path-conservative schemes have known deficiencies, which have been studied in detail in [4, 26].

In this paper we provide the first implementation of high order pathconservative schemes for non-conservative systems of the type (1) using the ADER approach together with space-time Adaptive Mesh Refinement (AMR). In this respect, the present work can be considered an extension of the method proposed in [42], where an ADER-WENO AMR scheme was proposed for the conservative case. ADER schemes, originally developed by E.F. Toro and collaborators in [89] and extensively used in the context of hyperbolic problems [85, 83, 37, 9, 11, are a class of numerical methods that obtain high order one-step schemes in time without the use of backward time levels, like in Adams-Bashforth type time integrators, and also without the use of substages, as used inside Runge-Kutta time integrators. The feature of high order one-step time integration will be actually the key for the construction of reasonably simple high order accurate AMR schemes together with time accurate local time stepping (LTS). For previous works including LTS see, e.g., [48, 38, 81, 63, 50, 92, 22, 58, 42.

However, the original ADER approach as proposed in [89, 85, 83] suffers from the drawback that it uses Taylor expansions in time where time derivatives are then replaced by spatial derivatives through a repeated use of the governing system of equations. This procedure, also known as the Cauchy-Kowalewski procedure, becomes rather cumbersome when dealing with complex systems of equations, and it fails completely in the presence of stiff source terms. A successful alternative to this strategy, that we also 
follow in this work, was proposed in [35]. There, a different formulation of the ADER approach was developed, where the Taylor series expansions and the Cauchy-Kovalewski procedure have been replaced by a local space-time Galerkin method, i.e. by a weak formulation of the PDE in space-time, see also the references [33, 41, 9, 11] where this new version of the ADER approach has been used.

As for the AMR aspects of our work, we have followed a cell-by-cell refinement strategy, which is particularly convenient within our high order one-step finite volume approach. Various examples of AMR schemes can be found in literature, for an overview see the original work by Berger et al. [17, 15, 14, 16, 13] and other implementations in [8, 10, 56, 70, 71, 76, 21, 82, 59, 95, 43].

The outline of the paper is as follows: In Sect. 2 we present our numerical method, with some emphasis on the implementation of path-conservative schemes within the ADER approach. Sect. 3 is devoted to the description of the Adaptive Mesh Refinement infrastructure, while in Sect. 4 the BaerNunziato equations are recalled. Sect. 5 contains a set of numerical test problems and computational results to validate the proposed high order pathconservative ADER-WENO AMR schemes, and, finally, Sect. 6reports some conclusions of our work and possible future extensions.

\section{Numerical method}

In order to obtain a numerical solution of the problem (1) we use higher order finite volume methods in the context of the ADER framework. To simplify the presentation, we first describe all details of the algorithm for a uniform Cartesian grid. The AMR technique will be described later. We recall that within the finite volume methodology, the numerical solution of the evolved quantities is represented at the beginning of each time-step by piecewise constant cell averages. The update of these data and the computation of the corresponding numerical fluxes can be performed using higher order piecewise polynomials of degree $M$ that have to be reconstructed, starting from the underlying piecewise constant cell averages, see [51, 54, 84].

\subsection{The Finite Volume scheme for nonconservative systems}

The system (1) is written in Cartesian coordinates and in three space dimensions as

$$
\frac{\partial \mathbf{u}}{\partial t}+\frac{\partial \mathbf{f}}{\partial x}+\frac{\partial \mathbf{g}}{\partial y}+\frac{\partial \mathbf{h}}{\partial z}+\mathbf{B}_{1} \frac{\partial \mathbf{u}}{\partial x}+\mathbf{B}_{2} \frac{\partial \mathbf{u}}{\partial y}+\mathbf{B}_{3} \frac{\partial \mathbf{u}}{\partial z}=\mathbf{S}(\mathbf{u}) .
$$


In order to obtain the finite volume representation of (3), we discretize the computational domain $\Omega$ in space-time control volumes defined as $\mathcal{I}_{i j k}=$ $I_{i j k} \times\left[t^{n}, t^{n}+\Delta t\right]=\left[x_{i-\frac{1}{2}}, x_{i+\frac{1}{2}}\right] \times\left[y_{j-\frac{1}{2}}, y_{j+\frac{1}{2}}\right] \times\left[z_{k-\frac{1}{2}}, z_{k+\frac{1}{2}}\right] \times\left[t^{n}, t^{n}+\Delta t\right]$, with $\Delta x_{i}=x_{i+\frac{1}{2}}-x_{i-\frac{1}{2}}, \Delta y_{j}=y_{j+\frac{1}{2}}-y_{j-\frac{1}{2}}, \Delta z_{k}=z_{k+\frac{1}{2}}-z_{k-\frac{1}{2}}$ and $\Delta t=t^{n+1}-t^{n}$. Each space control volume $I_{i j k}$ defines a computational cell, which will be denoted so forth as $\mathcal{C}_{m}$, identified by its mono-index $m$, with $1<m<N_{e}$, where $N_{e}$ is the number of computational cells in the domain. After integration of (3) over a space-time control volume $\mathcal{I}_{i j k}$ one obtains the following finite volume formulation:

$$
\begin{aligned}
\overline{\mathbf{u}}_{i j k}^{n+1}=\overline{\mathbf{u}}_{i j k}^{n} & -\frac{\Delta t}{\Delta x_{i}}\left[\left(\mathbf{f}_{i+\frac{1}{2}, j, k}-\mathbf{f}_{i-\frac{1}{2}, j, k}\right)+\frac{1}{2}\left(D_{i+\frac{1}{2}, j, k}^{x}+D_{i-\frac{1}{2}, j, k}^{x}\right)\right] \\
& -\frac{\Delta t}{\Delta y_{j}}\left[\left(\mathbf{g}_{i, j+\frac{1}{2}, k}-\mathbf{g}_{i, j-\frac{1}{2}, k}\right)+\frac{1}{2}\left(D_{i, j+\frac{1}{2}, k}^{y}+D_{i, j-\frac{1}{2}, k}^{y}\right)\right] \\
& -\frac{\Delta t}{\Delta z_{k}}\left[\left(\mathbf{h}_{i, j, k+\frac{1}{2}}-\mathbf{h}_{i, j, k-\frac{1}{2}}\right)+\frac{1}{2}\left(D_{i, j, k+\frac{1}{2}}^{z}+D_{i, j, k-\frac{1}{2}}^{z}\right)\right] \\
& +\Delta t\left(\overline{\mathbf{S}}_{i j k}-\overline{\mathbf{P}}_{i j k}\right),
\end{aligned}
$$

where

$$
\overline{\mathbf{u}}_{i j k}^{n}=\frac{1}{\Delta x_{i}} \frac{1}{\Delta y_{j}} \frac{1}{\Delta z_{k}} \int_{x_{i-\frac{1}{2}}}^{x_{i+\frac{1}{2}}} \int_{y_{j-\frac{1}{2}}}^{y_{j+\frac{1}{2}}} \int_{z_{k-\frac{1}{2}}}^{z_{k+\frac{1}{2}}} \mathbf{u}\left(x, y, z, t^{n}\right) d z d y d x
$$

is the spatial average of the solution in the element $I_{i j k}$ at time $t^{n}$, while

$$
\begin{aligned}
& \mathbf{f}_{i+\frac{1}{2}, j k}=\frac{1}{\Delta t} \frac{1}{\Delta y_{j}} \frac{1}{\Delta z_{k}} \int_{t^{n}}^{t^{n+1}} \int_{y_{j-\frac{1}{2}} z_{k-\frac{1}{2}}}^{y_{j+\frac{1}{2}}} \int_{k+\frac{1}{2}}^{z_{k}} \tilde{\mathbf{f}}\left(\mathbf{q}_{h}^{-}\left(x_{i+\frac{1}{2}}, y, z, t\right), \mathbf{q}_{h}^{+}\left(x_{i+\frac{1}{2}}, y, z, t\right)\right) d z d y d t \\
& \mathbf{g}_{i, j+\frac{1}{2}, k}=\frac{1}{\Delta t} \frac{1}{\Delta x_{i}} \frac{1}{\Delta z_{k}} \int_{t^{n}}^{t^{n+1}} \int_{x_{i-\frac{1}{2}}^{x_{i+\frac{1}{2}}}}^{z_{k+\frac{1}{2}}} \int_{z_{k-\frac{1}{2}}^{2}}^{\mathbf{g}}\left(\mathbf{q}_{h}^{-}\left(x, y_{j+\frac{1}{2}}, z, t\right), \mathbf{q}_{h}^{+}\left(x, y_{j+\frac{1}{2}}, z, t\right)\right) d z d x d t \\
& \mathbf{h}_{i j, k+\frac{1}{2}}=\frac{1}{\Delta t} \frac{1}{\Delta x_{i}} \frac{1}{\Delta y_{j}} \int_{t^{n}}^{t^{n+1}} \int_{x_{i-\frac{1}{2}}}^{x_{i+\frac{1}{2}}} \int_{y_{j-\frac{1}{2}}}^{y_{j+\frac{1}{2}}} \tilde{\mathbf{h}}\left(\mathbf{q}_{h}^{-}\left(x, y, z_{k+\frac{1}{2}}, t\right), \mathbf{q}_{h}^{+}\left(x, y, z_{k+\frac{1}{2}}, t\right)\right) d y d x d t
\end{aligned}
$$


are the average fluxes along each Cartesian direction. Furthermore we have defined the space-time average of the smooth part of the non-conservative product as

$$
\overline{\mathbf{P}}_{i j k}=\frac{1}{\Delta t} \frac{1}{\Delta x_{i}} \frac{1}{\Delta y_{j}} \frac{1}{\Delta z_{k}} \int_{t^{n}}^{t^{n+1}} \int_{x_{i-\frac{1}{2}}}^{x_{i+\frac{1}{2}}} \int_{y_{j-\frac{1}{2}}}^{y_{j+\frac{1}{2}}} \int_{z_{k-\frac{1}{2}}}^{z_{k+\frac{1}{2}}} \mathbf{B}\left(\mathbf{q}_{h}\right) \cdot \nabla \mathbf{q}_{h} d z d y d x d t
$$

and the space-time averaged source term

$$
\overline{\mathbf{S}}_{i j k}=\frac{1}{\Delta t} \frac{1}{\Delta x_{i}} \frac{1}{\Delta y_{j}} \frac{1}{\Delta z_{k}} \int_{t^{n}}^{t^{n+1}} \int_{x_{i-\frac{1}{2}}}^{x_{i+\frac{1}{2}}} \int_{y_{j-\frac{1}{2}}}^{y_{j+\frac{1}{2}}} \int_{z_{k-\frac{1}{2}}}^{z_{k+\frac{1}{2}}} \mathbf{S}\left(\mathbf{q}_{h}(x, y, z, t)\right) d z d y d x d t .
$$

The terms $\mathbf{q}_{h}$ in Eq. (6)-10) are piecewise space-time polynomials of degree $M$ and represent the time-evolved reconstruction polynomials. To obtain the $\mathbf{q}_{h}$ first a WENO reconstruction polynomial $\mathbf{w}_{h}$ is obtained from the cell averages $\overline{\mathbf{u}}_{i j k}$ at time $t^{n}$ (see Sect.2.2) and subsequently the time evolution is carried out via a local space-time DG predictor as illustrated in Sect. 2.3. In order to integrate the non-conservative product we use the Dal MasoLe Floch-Murat theory (see [65]) where the non-smooth part of the nonconservative term is defined as a Borel measure. In this formulation we therefore also need to account for the jumps of $\mathbf{q}_{h}$ at the element boundaries

$$
\begin{aligned}
& D_{i+\frac{1}{2}, j, k}^{x}=\frac{1}{\Delta t} \frac{1}{\Delta y_{j}} \frac{1}{\Delta z_{k}} \int_{t^{n}}^{t^{n+1}} \int_{y_{j-\frac{1}{2}} y_{j+\frac{1}{2}}}^{z_{k+\frac{1}{2}}} \int_{z^{2}}^{z_{k+\frac{1}{2}}} \mathcal{D}_{1}\left(\mathbf{q}_{h}^{-}\left(x_{i+\frac{1}{2}}, y, z, t\right), \mathbf{q}_{h}^{+}\left(x_{i+\frac{1}{2}}, y, z, t\right)\right) d z d y d t \\
& D_{i, j+\frac{1}{2}, k}^{y}=\frac{1}{\Delta t} \frac{1}{\Delta x_{i}} \frac{1}{\Delta z_{k}} \int_{t^{n}}^{t^{n+1}} \int_{x_{i-\frac{1}{2}} z_{i+\frac{1}{2}}^{z_{k+\frac{1}{2}}}}^{z_{k+\frac{1}{2}}} \mathcal{D}_{2}\left(\mathbf{q}_{h}^{-}\left(x, y_{j+\frac{1}{2}}, z, t\right), \mathbf{q}_{h}^{+}\left(x, y_{j+\frac{1}{2}}, z, t\right)\right) d z d x d t \\
& D_{i, j, k+\frac{1}{2}}^{z}=\frac{1}{\Delta t} \frac{1}{\Delta x_{i}} \frac{1}{\Delta y_{j}} \int_{t^{n}}^{t^{n+1}} \int_{x_{i-\frac{1}{2}}}^{x_{i+\frac{1}{2}}} \int_{y_{j-\frac{1}{2}}}^{y_{j+\frac{1}{2}}} \mathcal{D}_{3}\left(\mathbf{q}_{h}^{-}\left(x, y, z_{k+\frac{1}{2}}, t\right), \mathbf{q}_{h}^{+}\left(x, y, z_{k+\frac{1}{2}}, t\right)\right) d y d x d t
\end{aligned}
$$


using the path integrals

$$
\mathcal{D}_{m}\left(\mathbf{q}_{h}^{-}, \mathbf{q}_{h}^{+}\right)=\int_{0}^{1} \mathbf{B}_{m}\left(\Psi\left(\mathbf{q}_{h}^{-}, \mathbf{q}_{h}^{+}, s\right)\right) \frac{\partial \Psi}{\partial s} d s
$$

where $\Psi(s)$ is a path joining the left and right boundary extrapolated states $\mathbf{q}_{h}^{-}$and $\mathbf{q}_{h}^{+}$in state space. The simplest option is to use a straight-line segment path

$$
\Psi=\Psi\left(\mathbf{q}_{h}^{-}, \mathbf{q}_{h}^{+}, s\right)=\mathbf{q}_{h}^{-}+s\left(\mathbf{q}_{h}^{+}-\mathbf{q}_{h}^{-}\right), \quad 0 \leq s \leq 1 .
$$

Though simplified, this particular choice is useful in many applications since in the case of the shallow water equations it guarantees that the resulting numerical scheme is well-balanced and for the Baer-Nunziato model it has been shown to preserve the Abgrall condition [1, 2] when used with FORCE and Osher-type Riemann solvers [36, 40]. This may be no longer the case for other systems of equations, for which eventually more sophisticated paths must be adopted, see e.g. 68]. With the choice of the path (13), the terms $\mathcal{D}_{m}$ in 12 can be computed as

$$
\mathcal{D}_{m}\left(\mathbf{q}_{h}^{-}, \mathbf{q}_{h}^{+}\right)=\left(\int_{0}^{1} \mathbf{B}_{m}\left(\Psi\left(\mathbf{q}_{h}^{-}, \mathbf{q}_{h}^{+}, s\right)\right) d s\right)\left(\mathbf{q}_{h}^{+}-\mathbf{q}_{h}^{-}\right) .
$$

The practical computation of the integrals (14) is typically performed through a three-point Gauss-Legendre formula [36, 39, 40]. Finally, for the numerical approximation of the fluxes (6)-(8) we have either adopted a local LaxFriedrichs flux (Rusanov flux) or a simplified Osher-Solomon flux formula proposed in [39, 40, 60],

$$
\tilde{\mathbf{f}}\left(\mathbf{q}_{h}^{-}, \mathbf{q}_{h}^{+}\right)=\frac{1}{2}\left(\mathbf{f}\left(\mathbf{q}_{h}^{+}\right)+\mathbf{f}\left(\mathbf{q}_{h}^{-}\right)\right)+\frac{1}{2}\left(\int_{0}^{1}\left|\mathbf{A}_{1}(\Psi)\right| d s\right)\left(\mathbf{q}_{h}^{+}-\mathbf{q}_{h}^{-}\right),
$$

with

$$
\left|\mathbf{A}_{1}\right|=\mathbf{R}|\boldsymbol{\Lambda}| \mathbf{R}^{-1}, \quad|\boldsymbol{\Lambda}|=\operatorname{diag}\left(\left|\lambda_{1}\right|,\left|\lambda_{2}\right|, \ldots,\left|\lambda_{N}\right|\right),
$$

and where the path $\Psi$ in Eq. (15) is the same segment path adopted in (13) for the computation of the jumps $\mathcal{D}_{m}$. Again, the path integral is evaluated numerically using Gauss-Legendre quadrature rules. An entirely analogous procedure allows the computation of the numerical fluxes $\tilde{\mathbf{g}}$ and $\tilde{\mathbf{h}}$. Note that in the formulation above the numerical flux (15) contains in its dissipative term both, the conservative and the non-conservative part of the system (1). 


\subsection{WENO reconstruction}

In order to compute high order intercell fluxes and to integrate the source terms, it is necessary to carry out a reconstruction from the cell averages of the solution available at the beginning of each time step. To this extent, we provide the basic information about the WENO implementation that we have adopted, which differs from the standard one by [54]. Although a genuine multidimensional reconstruction is very natural within finite volume methods and is applicable to grids with arbitrary triangulations, we have adopted a cheaper dimension-by-dimension methodology [84, 85], which is also much simpler to implement in the presence of adaptive mesh refinement.

A crucial aspect to consider is the choice of the basis functions to be used in the reconstruction process. Two alternative options are available: a modal basis or a nodal basis, both of them rescaled on a reference unit interval, e.g. $I=[0 ; 1]$, through the following changes of coordinates valid for each element $I_{i j k}$

$$
\begin{aligned}
\xi & =\xi(x, i)=\frac{1}{\Delta x_{i}}\left(x-x_{i-\frac{1}{2}}\right), \\
\eta & =\eta(y, j)=\frac{1}{\Delta y_{j}}\left(y-y_{j-\frac{1}{2}}\right), \\
\zeta & =\zeta(z, k)=\frac{1}{\Delta z_{k}}\left(z-z_{k-\frac{1}{2}}\right) .
\end{aligned}
$$

The modal basis is formed by a set of $M+1$ linearly independent polynomials, typically the Legendre polynomials, having degree from zero to $M$. The nodal basis is instead formed by a set of $M+1$ linearly independent polynomials, $\left\{\psi_{l}\right\}_{l=1}^{M+1}$, all of degree $M$, which are effectively the Lagrange polynomials interpolating a set of $M+1$ nodal points, $\left\{x_{k}\right\}_{k=1}^{M+1}$, in such a way that

$$
\psi_{l}\left(x_{k}\right)=\delta_{l k} \quad l, k=1,2, \ldots, M+1 .
$$

Numerical experiments carried out by [53] have shown that it is more efficient to use the nodal basis instead of the modal one, especially if the GaussLegendre nodes are used. The reconstruction is performed for each cell $I_{i j k}$ on a reconstruction stencil that, for each Cartesian direction, is given by

$$
\mathcal{S}_{i j k}^{s, x}=\bigcup_{e=i-L}^{i+R} I_{e j k}, \quad \mathcal{S}_{i j k}^{s, y}=\bigcup_{e=j-L}^{j+R} I_{i e k}, \quad \mathcal{S}_{i j k}^{s, z}=\bigcup_{e=k-L}^{k+R} I_{i j e}
$$


where $L$ and $R$, which depend both on the order and on the specific stencil considered, are the spatial extension of the stencil to the left and to the right, respectively. Odd order schemes (even polynomial degrees $M$ ) always adopt three stencils, one central stencil $(s=1, L=R=M / 2)$, one fully left-sided stencil ( $s=2, L=M, R=0$ ) and one fully right-sided stencil ( $s=3, L=0$, $R=M)$. Even order schemes (odd polynomial degree $M$ ) always adopt four stencils, two of which are central $(s=0, L=$ floor $(M / 2)+1, R=$ floor $(M / 2))$ and $(s=1, L=$ floor $(M / 2), R=$ floor $(M / 2)+1)$, while the remaining two are again given by the fully left-sided and by the fully right-sided stencil, as defined before. The total amount of cells of each stencil is the same as that of the order of the scheme, namely $M+1$.

\subsubsection{Reconstruction along $x$}

The reconstruction is first performed in the $x$ direction, by writing the reconstructed polynomial in terms of the nodal basis $\psi_{l}(\xi)$

$$
\mathbf{w}_{h}^{s, x}\left(x, t^{n}\right)=\sum_{p=0}^{M} \psi_{p}(\xi) \hat{\mathbf{w}}_{i j k, p}^{n, s}:=\psi_{p}(\xi) \hat{\mathbf{w}}_{i j k, p}^{n, s},
$$

where we have used the Einstein summation convention, implying summation over indices appearing twice. Integral conservation on all elements of the stencil then yields the linear equation system from which the unknown reconstruction coefficients $\hat{\mathbf{w}}_{i j k, p}^{n, s}$ can be determined:

$$
\frac{1}{\Delta x_{e}} \int_{x_{e-\frac{1}{2}}}^{x_{e+\frac{1}{2}}} \psi_{p}(\xi(x)) \hat{\mathbf{w}}_{i j k, p}^{n, s} d x=\overline{\mathbf{u}}_{e j k}^{n}, \quad \forall I_{e j k} \in \mathcal{S}_{i j k}^{s, x} .
$$

Once the reconstruction has been performed for each of the stencils relative to the element $I_{i j k}$, we finally construct a data-dependent nonlinear combination of the polynomials obtained for each stencil, i.e.

$$
\mathbf{w}_{h}^{x}\left(x, t^{n}\right)=\psi_{p}(\xi) \hat{\mathbf{w}}_{i j k, p}^{n}, \quad \text { with } \quad \hat{\mathbf{w}}_{i j k, p}^{n}=\sum_{s=1}^{N_{s}} \omega_{s} \hat{\mathbf{w}}_{i j k, p}^{n, s},
$$

where, as specified at the beginning of Sect. 2.2, the number of stencils is $N_{s}=3$ or $N_{s}=4$, for even or odd $M$, respectively, while the nonlinear weights are given by the relations

$$
\omega_{s}=\frac{\tilde{\omega}_{s}}{\sum_{q} \tilde{\omega}_{q}}, \quad \tilde{\omega}_{s}=\frac{\lambda_{s}}{\left(\sigma_{s}+\epsilon\right)^{r}} .
$$


The oscillation indicator $\sigma_{s}$ is

$$
\sigma_{s}=\Sigma_{l m} \hat{\mathbf{w}}_{l}^{n, s} \hat{\mathbf{w}}_{m}^{n, s}
$$

and it requires the computation of the oscillation indicator matrix (see [35])

$$
\Sigma_{l m}=\sum_{\alpha=1}^{M} \int_{0}^{1} \frac{\partial^{\alpha} \psi_{l}(\xi)}{\partial \xi^{\alpha}} \cdot \frac{\partial^{\alpha} \psi_{m}(\xi)}{\partial \xi^{\alpha}} d \xi
$$

Unlike the original pointwise WENO of [54], which is $2 M+1$ order accurate in smooth regions of the solution, our $M+1$ order accurate implementation of WENO allows for a pragmatic choice of the coefficients $\lambda_{s}$. In particular, we select $\lambda_{s}=1$ for the one-sided stencils and $\lambda_{s}=10^{5}$ for the central stencils. Moreover, we use $\epsilon=10^{-14}$ and $r=8$.

\subsubsection{Reconstruction along $y$ and $z$}

Because the resulting reconstructed polynomial $\mathbf{w}_{h}^{x}\left(x, t^{n}\right)$ is only a polynomial in $x$ direction, but still an average in the $y$ and $z$ directions, the reconstruction algorithm described above must be applied again along the remaining two directions. In practice, the steps from (22) to (24) are repeated and details about this procedure can be found in [42].

\subsection{The local space-time Galerkin predictor}

The objective of the local space-time Galerkin predictor method is to provide the time evolution, locally for each element, of the reconstructed polynomials $\mathbf{w}_{h}\left(\mathbf{x}, t^{n}\right)$ obtained through the WENO reconstruction described before. However, unlike the original ADER approach of Titarev and Toro, which was based on a Taylor expansion in time and required a repeated use of the governing PDE system in order to substitute time derivatives with space derivatives, the new method relies on a weak integral formulation of the governing PDE in space-time using an element-local space-time Galerkin method. As a result, all that is required is a point-wise evaluation of fluxes and source terms. The result of the local space-time Galerkin predictor are the high order space-time polynomials $\mathbf{q}_{h}$ that are needed for the evaluation of the numerical fluxes, source terms and nonconservative jump terms in the scheme (4). In the following we briefly illustrate the method, referring to [35, 33, 41, 36, 53, 42] for more details. 
After introducing the reference time coordinate $\tau=\left(t-t^{n}\right) / \Delta t$, we write the system $(1)$ in terms of the reference coordinates $\tau$ and $\vec{\xi}=(\xi, \eta, \zeta)$, see the definitions (17)-19), to get

$$
\frac{\partial \mathbf{u}}{\partial \tau}+\frac{\partial \mathbf{f}^{*}}{\partial \xi}+\frac{\partial \mathbf{g}^{*}}{\partial \eta}+\frac{\partial \mathbf{h}^{*}}{\partial \zeta}+\mathbf{B}_{1}^{*} \frac{\partial \mathbf{u}}{\partial \xi}+\mathbf{B}_{2}^{*} \frac{\partial \mathbf{u}}{\partial \eta}+\mathbf{B}_{3}^{*} \frac{\partial \mathbf{u}}{\partial \zeta}=\mathbf{S}^{*}
$$

with

$$
\mathbf{f}^{*}=\frac{\Delta t}{\Delta x_{i}} \mathbf{f}, \quad \mathbf{g}^{*}=\frac{\Delta t}{\Delta y_{j}} \mathbf{g}, \quad \mathbf{h}^{*}=\frac{\Delta t}{\Delta z_{k}} \mathbf{h}
$$

and

$$
\mathbf{B}_{1}^{*}=\frac{\Delta t}{\Delta x_{i}} \mathbf{B}_{1}, \quad \mathbf{B}_{2}^{*}=\frac{\Delta t}{\Delta y_{j}} \mathbf{B}_{2}, \quad \mathbf{B}_{3}^{*}=\frac{\Delta t}{\Delta z_{k}} \mathbf{B}_{3}, \quad \mathbf{S}^{*}=\Delta t \mathbf{S} .
$$

To obtain a local space-time discontinuous Galerkin approach, we multiply expression (28) by piecewise space-time polynomials $\theta_{\mathfrak{q}}(\xi, \eta, \zeta, \tau)$ of degree $M$, which are given by a tensor-product of the basis functions $\psi_{l}$ adopted in the reconstruction procedure. Here, we use the multi-index $\mathfrak{q}=(p, q, r, s)$. Integration over the space-time reference control volume then yields

$$
\begin{aligned}
& \int_{0}^{1} \int_{0}^{1} \int_{0}^{1} \int_{0}^{1} \theta_{\mathfrak{q}}\left(\frac{\partial \mathbf{q}_{h}}{\partial \tau}+\frac{\partial \mathbf{f}^{*}}{\partial \xi}+\frac{\partial \mathbf{g}^{*}}{\partial \eta}+\frac{\partial \mathbf{h}^{*}}{\partial \zeta}\right) d \xi d \eta d \zeta d \tau= \\
& \int_{0}^{1} \int_{0}^{1} \int_{0}^{1} \int_{0}^{1} \theta_{\mathfrak{q}}\left(\mathbf{S}^{*}-\mathbf{B}_{1}^{*} \frac{\partial \mathbf{q}_{h}}{\partial \xi}-\mathbf{B}_{2}^{*} \frac{\partial \mathbf{q}_{h}}{\partial \eta}-\mathbf{B}_{3}^{*} \frac{\partial \mathbf{q}_{h}}{\partial \zeta}\right) d \xi d \eta d \zeta d \tau,
\end{aligned}
$$

The discrete space-time solution of Eq. (31) will be henceforth denoted by $\mathbf{q}_{h}$, and is expanded over the same space-time basis of polynomials as

$$
\mathbf{q}_{h}(\vec{\xi}, \tau)=\theta_{\mathfrak{p}}(\vec{\xi}, \tau) \hat{\mathbf{q}}_{\mathfrak{p}}
$$

where $\hat{\mathbf{q}}_{\mathfrak{p}}$ are the unknown nodal degrees of freedom. A similar nodal representation is provided for the remaining terms entering Eq. (31). Integration by parts in time yields

$$
\int_{0}^{1} \int_{0}^{1} \int_{0}^{1} \theta_{\mathfrak{q}}(\vec{\xi}, 1) \theta_{\mathfrak{p}}(\vec{\xi}, 1) \hat{\mathbf{q}}_{\mathfrak{p}} d \xi d \eta d \zeta-\int_{0}^{1} \int_{0}^{1} \int_{0}^{1} \int_{0}^{1}\left(\frac{\partial}{\partial \tau} \theta_{\mathfrak{q}}\right) \theta_{\mathfrak{p}} \hat{\mathbf{q}}_{\mathfrak{p}} d \xi d \eta d \zeta d \tau=
$$




$$
\begin{aligned}
& \int_{0}^{1} \int_{0}^{1} \int_{0}^{1} \theta_{\mathfrak{q}}(\vec{\xi}, 0) \mathbf{w}_{h}\left(\vec{\xi}, t^{n}\right) d \xi d \eta d \zeta \\
& -\int_{0}^{1} \int_{0}^{1} \int_{0}^{1} \int_{0}^{1} \theta_{\mathfrak{q}}\left(\frac{\partial}{\partial \xi} \mathbf{f}^{*}\left(\mathbf{q}_{h}\right)+\frac{\partial}{\partial \eta} \mathbf{g}^{*}\left(\mathbf{q}_{h}\right)+\frac{\partial}{\partial \zeta} \mathbf{h}^{*}\left(\mathbf{q}_{h}\right)\right) d \xi d \eta d \zeta d \tau \\
& +\int_{0}^{1} \int_{0}^{1} \int_{0}^{1} \int_{0}^{1} \theta_{\mathfrak{q}}\left(\mathbf{S}^{*}-\mathbf{B}_{1}^{*} \frac{\partial \mathbf{q}_{h}}{\partial \xi}-\mathbf{B}_{2}^{*} \frac{\partial \mathbf{q}_{h}}{\partial \eta}-\mathbf{B}_{3}^{*} \frac{\partial \mathbf{q}_{h}}{\partial \zeta}\right) d \xi d \eta d \zeta d \tau,
\end{aligned}
$$

Eq. (33) should be regarded as a nonlinear algebraic equation, to be solved locally for each element of the computational grid in the unknowns $\hat{\mathbf{q}}_{\mathbf{p}}$. Additional details about the local space-time Galerkin predictor for the specific case of non-conservative systems can be found for example in [36]. We emphasize that the choice of a nodal basis based on Gauss-Legendre nodes allows a dimension-by-dimension evaluation of the terms appearing in Eq. (33).

\section{Adaptive Mesh Refinement}

A detailed illustration of the AMR implementation within our ADERWENO approach has been presented in [42]. The description we provide here is self-contained, but focused on the essential aspects only. Unlike the original patch-based block-structured approach by Berger \& Oliger [17, 15, 14], we refine individual Cartesian cells, which are treated as elements of a tree data structure, like in [57]. This choice is particularly suited to our element-local space-time DG predictor, which does not need any exchange of information through neighbor elements, and can therefore be implemented with no modifications even if two adjacent cells belong to two different levels of grid refinement.

\subsection{AMR implementation}

Each level of refinement is indicated with $\ell$, ranging from the coarsest level $\ell=0$ to the maximally refined level $\ell=\ell_{\max }$, beyond which no further refinement is possible. In addition, we use $\mathcal{L}_{\ell}$ to denote the union of all elements up to level $\ell$. Any cell $\mathcal{C}_{m}$, at any level of refinement, is identified with a unique positive integer number $m$, with $1 \leq m \leq N_{\text {Cells }}$, where $N_{\text {Cells }}$ is the (time-dependent) total number of cells at any given time. When a cell $\mathcal{C}_{m}$ at level $\ell$ is refined, we refer to it as a mother or parent cell, and 
the cells on the next refinement level $\ell+1$ contained in it are called children cells. Moreover, the Neumann neighbors $\mathcal{N}_{m}$ of a cell $\mathcal{C}_{m}$ are the neighbor cells that share a face with $\mathcal{C}_{m}$. Each cell has $2 d$ Neumann neighbors in $d$ space dimensions, except of course the case of the cells at the boundaries of the computational domain. On the other hand, the Voronoi neighbors $\mathcal{V}_{m}$ of a cell $\mathcal{C}_{m}$ are those cells which share at least one node with $\mathcal{C}_{m}$, and each cell has $3^{d}-1$ Voronoi neighbors in $d$ space dimensions.

Since the high order finite volume schemes used in this paper need information from neighbors to carry out the WENO reconstruction (even more than just the Voronoi neighbors $\mathcal{V}_{m}$ ), each refined cell on a level $\ell+1$ must be surrounded by a layer of either real or virtual cells on the same level. The layer thickness must be greater or equal to the size of the reconstruction stencil. Likewise, a mother cell on level $\ell$ that is refined continues to exist as a virtual mother cell since it may be surrounded by non-refined cells on the same level which need its information for reconstruction. A schematic representation of this mechanism involving one level of refinement is reported in figure 1. There, the central cell of level $\ell$ is refined, hence it becomes virtual and has real children on level $\ell+1$, while the surrounding cells are virtually refined in order to allow the real cells on level $\ell+1$ to perform the WENO reconstruction.

Having introduced this terminology, it is convenient to list schematically the rules that are adopted in our cell-by-cell AMR implementation.

- Any AMR scheme requires a criterion for deciding whether a given cell $\mathcal{C}_{m}$ needs refinement or recoarsening. We have adopted the same strategy described in [62, which is based on the calculation of a second derivative error. A cell $\mathcal{C}_{m}$ is marked for refinement if $\chi_{m}>\chi_{\text {ref }}$, while it is marked for recoarsening if $\chi_{m}<\chi_{\text {rec }}$, where

$$
\chi_{m}=\sqrt{\frac{\sum_{k, l}\left(\partial^{2} \Phi / \partial x_{k} \partial x_{l}\right)^{2}}{\sum_{k, l}\left[\left(\left|\partial \Phi / \partial x_{k}\right|_{i+1}+\left|\partial \Phi / \partial x_{k}\right|_{i}\right) / \Delta x_{l}+\varepsilon\left|\left(\partial^{2} / \partial x_{k} \partial x_{l}\right)\right||\Phi|\right]^{2}}} .
$$

The summation $\sum_{k, l}$ is taken over the number of space dimension of the problem in order to include the cross term derivatives, whereas $\Phi=\Phi(\mathbf{u})$ is a generic indicator function of the conservative variables u. In most cases we have adopted $\chi_{\text {ref }}$ in the range $\sim[0.2,0.25]$ and $\chi_{\text {rec }}$ in the range $\sim[0.05,0.15]$. Finally, the parameter $\varepsilon$ acts as a filter which prevents refinement in regions of small ripples and is given the 


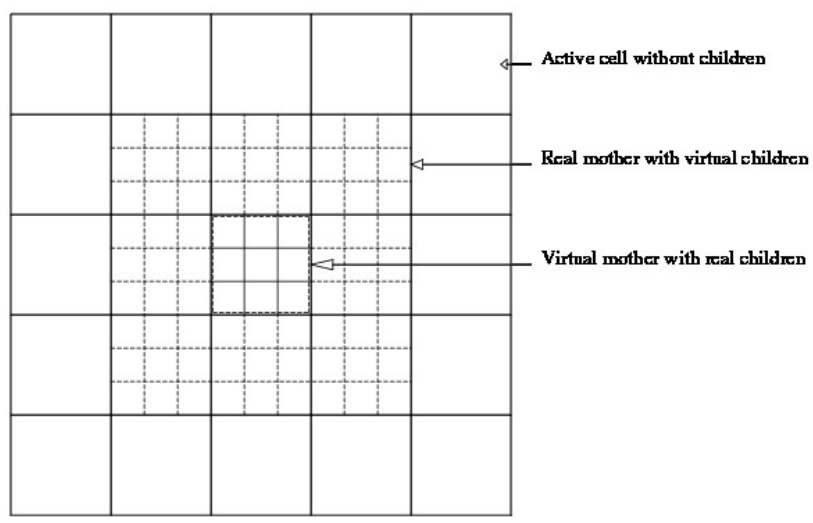

Figure 1: Sketch of a cell-by-cell refinement of the central cell $\mathcal{C}_{m}$ on level $\ell$. The children of $\mathcal{C}_{m}$ are real cells on level $\ell+1$, surrounded by virtual children of the Voronoi neighbors $\mathcal{V}_{m}$ of cell $\mathcal{C}_{m}$. The virtual children are on the same level $\ell+1$ and are needed for reconstruction.

value $\varepsilon=0.01$.

- Whenever a mother cell of the level $\ell$ is refined, it generates $\mathfrak{r}^{d}$ children cells, such that

$$
\Delta x_{\ell}=\mathfrak{r} \Delta x_{\ell+1} \quad \Delta y_{\ell}=\mathfrak{r} \Delta y_{\ell+1} \quad \Delta z_{\ell}=\mathfrak{r} \Delta z_{\ell+1} .
$$

In addition, and as commented below, the time steps are also chosen locally on each level so that

$$
\Delta t_{\ell}=\mathfrak{r} \Delta t_{\ell+1}
$$

Due to the high order WENO reconstruction, the refinement factor $\mathfrak{r}$ must satisfy $\mathfrak{r} \geq M$.

- At any level of refinement, each cell $\mathcal{C}_{m}$ has one among three possible status flags, which we denote by $\sigma$. The first possibility is that $\mathcal{C}_{m}$ is an active cell $(\sigma=0)$, in which case it is updated through the finite volume scheme described in the previous Section 2. The second possibility is that $\mathcal{C}_{m}$ is a virtual child cell $(\sigma=1)$ and is updated by projection 
of the mother's high order space-time polynomial. In practice, the virtual children receive their values from the active mother via standard $L_{2}$ projection. For this purpose, the space-time polynomials $\mathbf{q}_{h}$ can be conveniently evaluated at any time. This operation is needed for performing the reconstruction on the finer grid level at intermediate times. The projection operator for a cell $\mathcal{C}_{m}$ on level $\ell$ is simply given by evaluating the space-time polynomial $\mathbf{q}_{h}$ of its mother at any given time $t_{\ell}^{n}$ as follows:

$$
\overline{\mathbf{u}}_{m}\left(t_{\ell}^{n}\right)=\frac{1}{\Delta x_{\ell}} \frac{1}{\Delta y_{\ell}} \frac{1}{\Delta z_{\ell}} \int_{\mathcal{C}_{m}} \mathbf{q}_{h}\left(\mathbf{x}, t_{\ell}^{n}\right) d \mathbf{x} .
$$

Finally, $\mathcal{C}_{m}$ can be a virtual mother cell $(\sigma=-1)$, updated by recursively averaging over all children from higher refinement levels. Namely, the virtual mother cell obtains its cell average by averaging recursively over the cell averages of all its children, namely including the possible children of their children. If we denote the set of children of a cell $\mathcal{C}_{m}$ by $\mathcal{B}_{m}$, then the averaging operator is given by

$$
\overline{\mathbf{u}}_{m}=\frac{1}{\mathfrak{r}^{d}} \sum_{\mathcal{C}_{k} \in \mathcal{B}_{m}} \overline{\mathbf{u}}_{k}
$$

- Only real cells $(\sigma=0)$ can be refined. Therefore, if a virtual cell needs to be refined it must be first activated.

- The levels of refinement of two cells that are Voronoi neighbors of each other can only differ by at most unity. Moreover, every cell has Voronoi neighbors, which can be either active or virtual, at the same level of refinement.

\subsection{AMR local time stepping}

As anticipated above, each of the refinement levels is advanced in time with its own local time-step, i.e. $\Delta t_{\ell}=\mathfrak{r} \Delta t_{\ell+1}$. The use of time steps that are integer multiples of each other among subsequent levels is very convenient, and indeed very natural within AMR. However, alternative local time stepping schemes are also possible (see [38, 81, 63]), where a different local time step is allowed for each element. After denoting by $t_{\ell}^{n}$ and $t_{\ell}^{n+1}$ the 

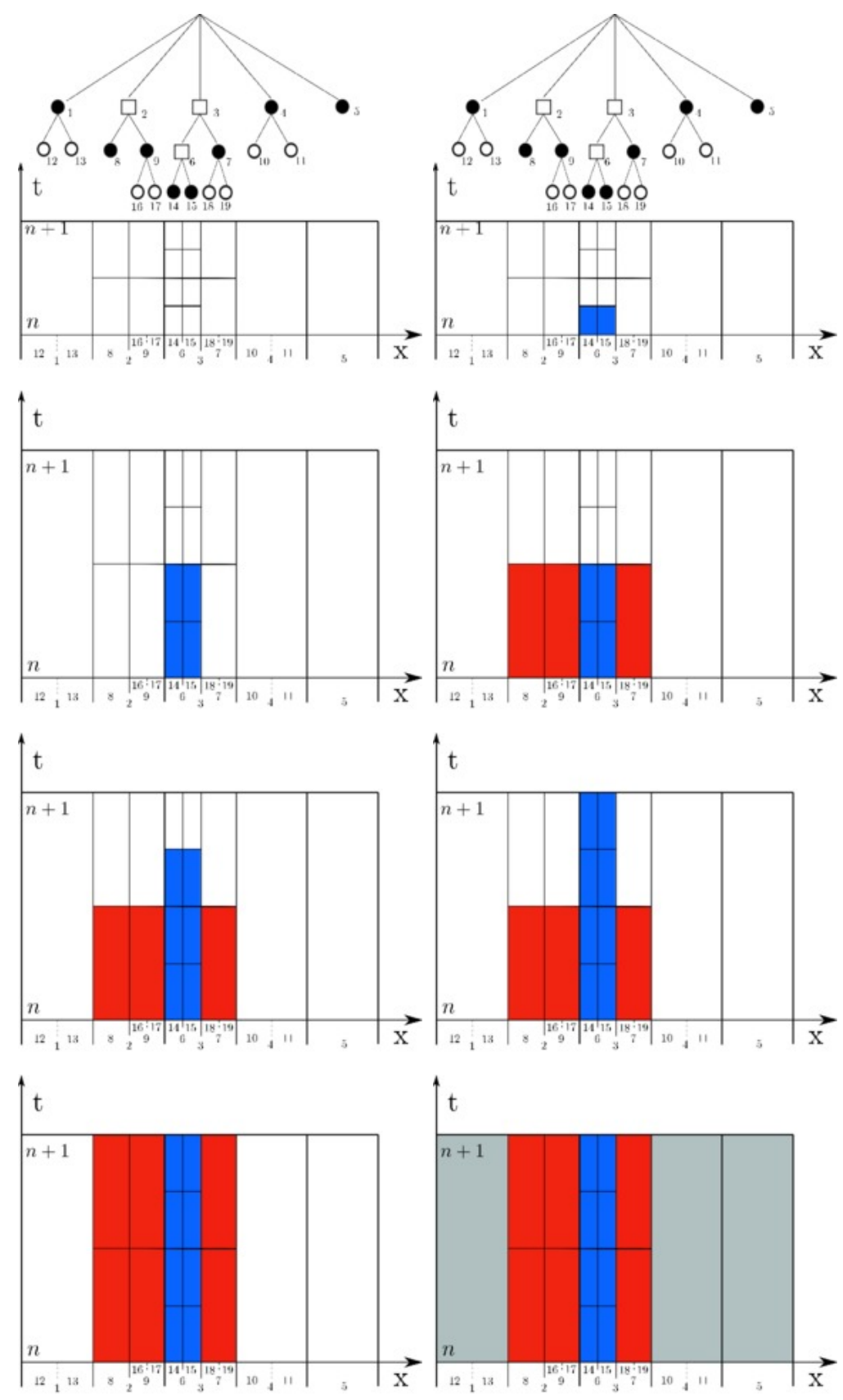

Figure 2: Example of a local time stepping algorithm involving two levels of refinement with $\mathfrak{r}=2$. Legend: $\bullet$ (regular active cell), $\bigcirc$ (virtual refined cell), $\square$ (virtual coarse cell). 
current and future times of the level $\ell$, the first level to be updated is the one with the largest value of $\ell$ satisfying the update criterion ${ }^{2}[38$

$$
t_{\ell}^{n+1} \leq t_{\ell-1}^{n+1}, \quad 0 \leq \ell \leq \ell_{\max } .
$$

In practice, starting from the common initial time $t=0$, the finest level of refinement $\ell_{\max }$ is evolved first and performs a number of $\mathfrak{r}$ sub-timesteps before the next coarser level $\ell_{\max }-1$ performs its first time update. This procedure is then applied recursively and it implies a total amount of $\mathfrak{r}^{\ell}$ sub-

timesteps on each level to be performed in order to reach the time $t_{0}^{n+1}$ of the coarsest level. As example of the application of the local time stepping strategy to a one dimensional case involving two levels of refinement with $\mathfrak{r}=2$ is reported in Fig, 2 ,

Thanks to the use of the local space-time predictor, which computes the predictor solution $\mathbf{q}_{h}$ for each element valid from time $t_{\ell}^{n}$ to time $t_{\ell}^{n+1}$, the computation of numerical fluxes between two adjacent cells on different levels of refinement is rather straightforward. Further details about the actual implementation of the local time stepping procedure and of the AMR parallelization through the standard Message Passing Interface (MPI) can be found in [42].

\section{The Baer-Nunziato equations}

A Baer-Nunziato type model for compressible two-phase flow without relaxation terms is given by the following system of equations, see [7, 78, 6, 80, 29, 69]:

$$
\begin{aligned}
& \frac{\partial}{\partial t}\left(\phi_{1} \rho_{1}\right)+\nabla \cdot\left(\phi_{1} \rho_{1} \mathbf{v}_{1}\right)=0 \\
& \frac{\partial}{\partial t}\left(\phi_{1} \rho_{1} \mathbf{v}_{1}\right)+\nabla \cdot\left(\phi_{1} \rho_{1} \mathbf{v}_{1} \mathbf{v}_{1}\right)+\nabla \phi_{1} p_{1}=p_{I} \nabla \phi_{1} \\
& \frac{\partial}{\partial t}\left(\phi_{1} \rho_{1} E_{1}\right)+\nabla \cdot\left(\left(\phi_{1} \rho_{1} E_{1}+\phi_{1} p_{1}\right) \mathbf{v}_{1}\right)=-p_{I} \partial_{t} \phi_{1} \\
& \frac{\partial}{\partial t}\left(\phi_{2} \rho_{2}\right)+\nabla \cdot\left(\phi_{2} \rho_{2} \mathbf{v}_{2}\right)=0 \\
& \frac{\partial}{\partial t}\left(\phi_{2} \rho_{2} \mathbf{v}_{2}\right)+\nabla \cdot\left(\phi_{2} \rho_{2} \mathbf{v}_{2} \mathbf{v}_{2}\right)+\nabla \phi_{2} p_{2}=p_{I} \nabla \phi_{2} \\
& \frac{\partial}{\partial t}\left(\phi_{2} \rho_{2} E_{2}\right)+\nabla \cdot\left(\left(\phi_{2} \rho_{2} E_{2}+\phi_{2} p_{2}\right) \mathbf{v}_{2}\right)=p_{I} \partial_{t} \phi_{1} \\
& \frac{\partial}{\partial t} \phi_{1}+\mathbf{v}_{I} \nabla \phi_{1}=0
\end{aligned}
$$

\footnotetext{
${ }^{2}$ We define $t_{-1}^{n+1}:=t_{0}^{n+1}$, so that also the scheduling of level $\ell=0$ is taken into account.
} 
The system is closed by the stiffened equation of state (EOS):

$$
e_{k}=\frac{p_{k}+\gamma_{k} \pi_{k}}{\rho_{k}\left(\gamma_{k}-1\right)}
$$

Here, $\phi_{k}$ denotes the volume fraction of phase $k, \rho_{k}$ is the density, $\mathbf{v}_{k}$ is the velocity vector, $E_{k}=e_{k}+\frac{1}{2} \mathbf{v}_{k}^{2}$ and $e_{k}$ are the phase specific total and internal energies, respectively. In the literature, one of the phases is often also called the solid phase and the other one the gas phase. Defining arbitrarily the first phase as the solid phase in the rest of the paper we will therefore use the subscripts 1 and $s$ as well as 2 and $g$ as synonyms. For the interface velocity $\mathbf{u}_{I}$ and pressure $p_{I}$ we choose $\mathbf{v}_{I}=\mathbf{v}_{1}$ and $p_{I}=p_{2}$, according to [7], although other choices are also possible (see e.g. the paper by Saurel and Abgrall [78]). The state vector $\mathbf{u}$ is

$$
\mathbf{u}=\left(\phi_{1} \rho_{1}, \phi_{1} \rho_{1} \mathbf{v}_{1}, \phi_{1} \rho_{1} E_{1}, \phi_{2} \rho_{2}, \phi_{2} \rho_{2} \mathbf{v}_{2}, \phi_{2} \rho_{2} E_{2}, \phi_{1}\right) .
$$

The system (40) can be cast in the form prescribed by (1) by collecting all the non-conservative terms in the matrix $\mathbf{B}(\mathbf{u})$, while keeping the conservative

part of the system expressed through $\mathbf{F}(\mathbf{u})$. An exhaustive treatment of the mathematical properties of the Baer-Nunziato equations can be found in [6, 29, 80, 87, where also exact and approximate solutions to the Riemann problem are given.

\section{Test Problems}

In all test problems shown below the indicator function for the refinement and recoarsening criterion has been chosen as

$$
\Phi=\sqrt{\left(\frac{\phi_{1} \rho_{1}}{\rho_{1,0}}\right)^{2}+\left(\frac{\phi_{2} \rho_{2}}{\rho_{2,0}}\right)^{2}},
$$

with some reference densities $\rho_{1,0}$ and $\rho_{2,0}$, respectively.

\subsection{Smooth vortex problem}

The first test that we have considered is given by a stationary and axisymmetric solution of the Baer-Nunziato equations and has first been reported in [36]. The resulting configuration describes a vortex-type solution with no radial motion. Because of these assumptions, the continuity and the energy 
equations are automatically satisfied. After choosing a simple dependence on the radius $r$ of the pressure and of the volume fraction of the solid phase, namely

$$
\begin{aligned}
& p_{k}=p_{k 0}\left(1-\frac{1}{4} e^{\left(1-r^{2} / s_{k}^{2}\right)}\right), \quad(k=1,2), \\
& \phi_{1}=\frac{1}{3}+\frac{1}{2 \sqrt{2 \pi}} e^{-r^{2} / 2}
\end{aligned}
$$

the momentum equations can be easily solved, to provide the velocity field of the vortex as

$$
\begin{aligned}
u_{1}^{\theta} & =\frac{1}{2 s_{1} D} \sqrt{r D\left[p_{10}\left(4 \sqrt{2 \pi} F_{1}+6 H_{1}-12 G s_{1}^{2}+3 H_{1} s_{1}^{2}\right)+3 p_{20} s_{1}^{2}\left(4 G-H_{2}\right)\right]} \\
u_{2}^{\theta} & =\frac{r \sqrt{2}}{2 \rho_{2} s_{2}} \sqrt{\rho_{2} p_{20} F_{2}}
\end{aligned}
$$

where

$$
H_{k}=e^{-\frac{2 r^{2}+r^{2} s_{k}^{2}-2 s_{k}^{2}}{2 s_{k}^{2}}}, \quad F_{k}=e^{-\frac{\left(r-s_{k}\right)\left(r+s_{k}\right)}{s_{k}^{2}}}, \quad(k=1,2),
$$

and

$$
G=e^{-r^{2} / 2}, \quad D=\rho_{1}(2 \sqrt{2 \pi}+3 G) .
$$

In order to make the test problem unsteady, the vortex is then boosted along the diagonal of the computational domain through a Galilean transformation of the velocity, with components $\bar{u}=\bar{v}$. In our tests we have chosen the following parameters

$$
\rho_{1}=1, \quad \rho_{2}=2, \quad p_{10}=1, \quad p_{20}=\frac{3}{2}, \quad s_{1}=\frac{3}{2}, \quad s_{2}=\frac{7}{5}, \quad \bar{u}=\bar{v}=2,
$$

while the computational domain is $\Omega=[-10 ; 10] \times[-10 ; 10]$ with four periodic boundary conditions, in such a way that the exact solution of the problem is given by the initial condition after $T=10$. In Table 1 we have reported the results of the convergence tests, where we have used the third and fourth order version of the method. Here $\rho_{1,0}=\rho_{2,0}=1$ have been chosen. The convergence rates have been computed with respect to an initially uniform mesh, as proposed by Berger and Oliger in [17]. 
Table 1: Numerical convergence results for the vortex test using the one-step ADERWENO finite volume scheme. The error norms refer to the variable $\rho_{1}$ at the final time $T=10$, and have been computed with $\ell_{\max }=2$. The asterisk ${ }^{*}$ refers to a uniform grid.

\begin{tabular}{lcclcc}
\hline$N_{G} \times N_{G}$ & $\epsilon_{L_{2}}$ & $\mathcal{O}\left(L_{2}\right)$ & $N_{G} \times N_{G}$ & $\epsilon_{L_{2}}$ & $\mathcal{O}\left(L_{2}\right)$ \\
\hline \multicolumn{5}{c}{$\mathcal{O} 3$} & $\mathcal{O} 4$ \\
\hline $15 \times 15^{*}$ & $4.9627 \mathrm{E}-01$ & & $15 \times 15^{*}$ & $4.6443 \mathrm{E}-01$ & \\
$30 \times 30$ & $2.5428 \mathrm{E}-02$ & 4.29 & $30 \times 30$ & $2.3166 \mathrm{E}-02$ & 4.33 \\
$45 \times 45$ & $1.3665 \mathrm{E}-02$ & 3.27 & $45 \times 45$ & $1.0674 \mathrm{E}-02$ & 3.43 \\
$60 \times 60$ & $7.8621 \mathrm{E}-03$ & 2.99 & $60 \times 60$ & $1.0115 \mathrm{E}-03$ & 4.42 \\
$90 \times 90$ & $2.0279 \mathrm{E}-03$ & 3.07 & $75 \times 75$ & $5.6484 \mathrm{E}-04$ & 4.17 \\
$120 \times 120$ & $9.9613 \mathrm{E}-04$ & 2.99 & $90 \times 90$ & $2.9489 \mathrm{E}-04$ & 4.11 \\
\hline
\end{tabular}
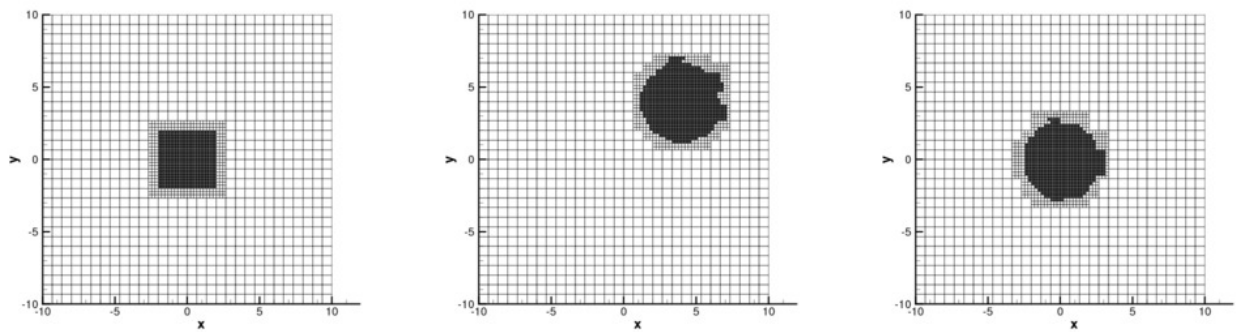

Figure 3: AMR grid of the isentropic-vortex test at the initial time (left panel), at time $t=2.0$ (central panel) and at the final time $t=10$ (right panel). Two levels of refinement have been adopted $\left(\ell_{\max }=2\right)$, starting from a uniform $45 \times 45$ grid.

\subsection{Riemann problems}

The high order space-time adaptive ADER-WENO methods proposed in this paper are particularly well suited for the accurate resolution of material interfaces in multi-fluid and multi-phase flow problems. This claim is validated in the following by solving a set of $1 \mathrm{D}$ shock tube problems on space-time adaptive Cartesian meshes in 2D. The exact solutions for the Riemann problems solved here have been provided in [6, 80, 29]. From these papers a set of six Riemann problems has been chosen, see Table 2. The same set of problems has already been solved with high order unstructured one-step WENO finite volume schemes using a centered path-conservative FORCE method in [36]. A subset of these Riemann problems has been 
solved again in [40] using the more accurate path-conservative extension of the Osher method, which is also used in this paper.

The two-dimensional computational domain is $\Omega=[-0.5 ; 0.5] \times[0 ; 1]$, which is discretized at the level 0 grid with only $100 \times 10$ cells. A maximum number of two refinement levels $\left(\ell_{\max }=2\right)$ is chosen, together with a refinement factor of $\mathfrak{r}=4$. The discontinuity is located initially at $x=0$ and the final simulation times are given in Table 2. For all test cases a third order ADER-WENO scheme is used with reconstruction in characteristic variables. In $x$-direction transmissive boundary conditions are imposed and periodic boundaries are applied in $y$-direction. The parameters for the refinement criterion are $\rho_{1,0}=\rho_{2,0}=1$ apart for RP2 and RP4, where $\rho_{1,0}=1000$ and $\rho_{2,0}=1$.

The results for the AMR computations are shown in Figs. 4-9, A sketch of the AMR grid at the final simulation time is depicted on the top left of each figure together with a cut through the reconstructed numerical solution $\mathbf{w}_{h}$ on 200 equidistant points along the $x$-axis in the remaining sub-figures. For RP4 the same quantities as in [80] are shown. For all six problems we obtain an excellent agreement between the high order AMR computations and the exact reference solutions provided in [6, 80, 29]. The agreement is much better than the one obtained in the previous publications of the authors [36, 40]. The solid contact is resolved perfectly well in all cases. Furthermore, no spurious post shock oscillations as reported in [36] for RP5 are visible in the present high order ADER-WENO simulations with AMR. Our results clearly confirm that the combination of adaptive mesh refinement (AMR) with high order WENO finite volume schemes with the little diffusive Osher-type Riemann solver [39, 40] is very well suited for the simulation of compressible multi-phase flows, as claimed at the beginning of this section.

\subsection{Explosion problems in multiple space dimensions}

An explosion problem for the Baer-Nunziato equations can be solved both in two and in three space dimensions, after setting-up the following initial conditions

$$
\mathbf{u}(\mathbf{x}, 0)=\left\{\begin{array}{lll}
\mathbf{u}_{i} & \text { if } \quad r \leq R \\
\mathbf{u}_{o} & \text { if } \quad r>R
\end{array}\right.
$$

where $\mathbf{x}$ is the vector of spatial coordinates, $r=\sqrt{\mathbf{x}^{2}}$, and $R$ is the radius of the initial discontinuity, which we have set equal to 0.5 and to 0.4 for the twodimensional and for the three-dimensional explosion tests, respectively. The 

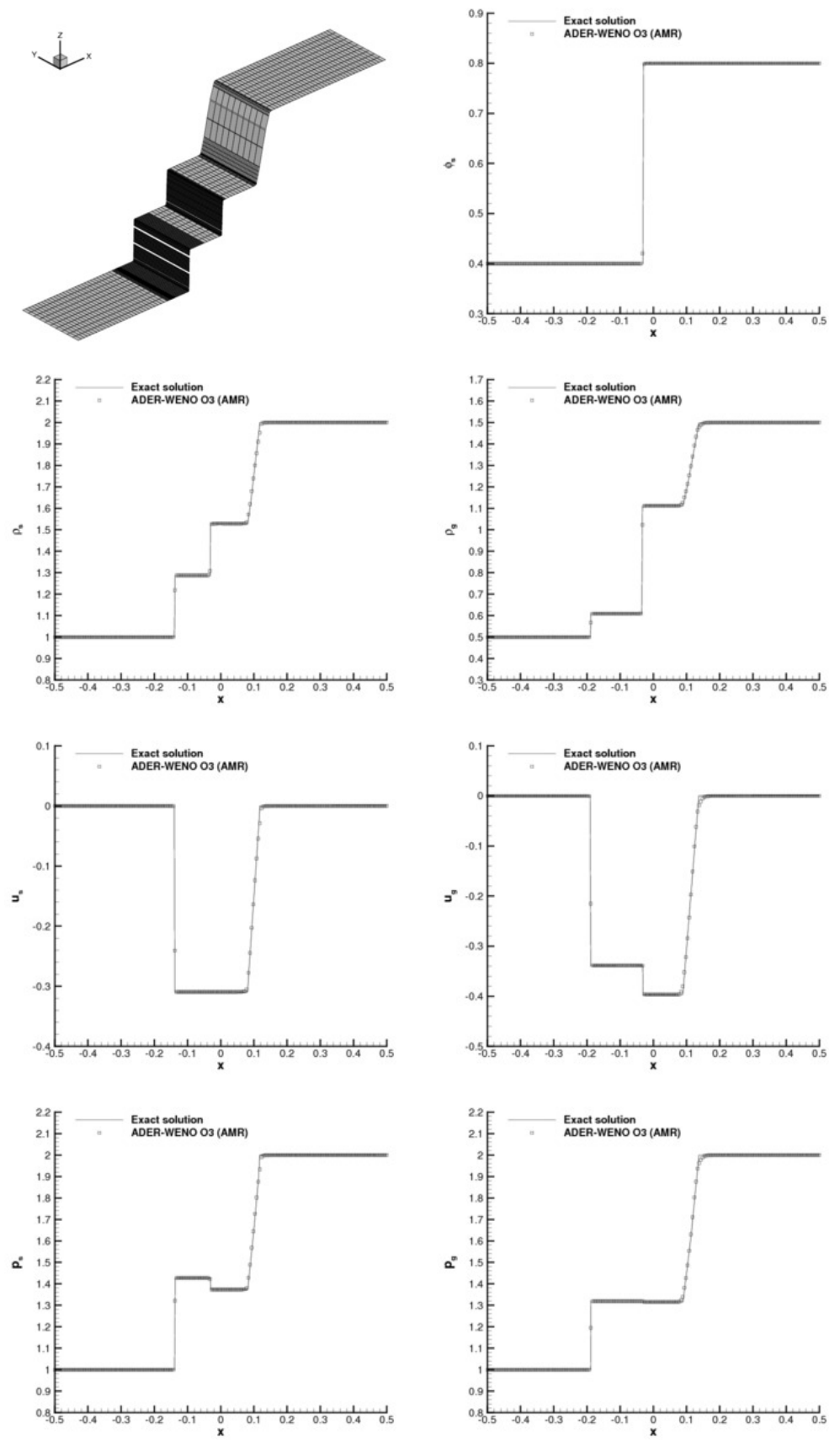

Figure 4: Results for Riemann problem RP1. 

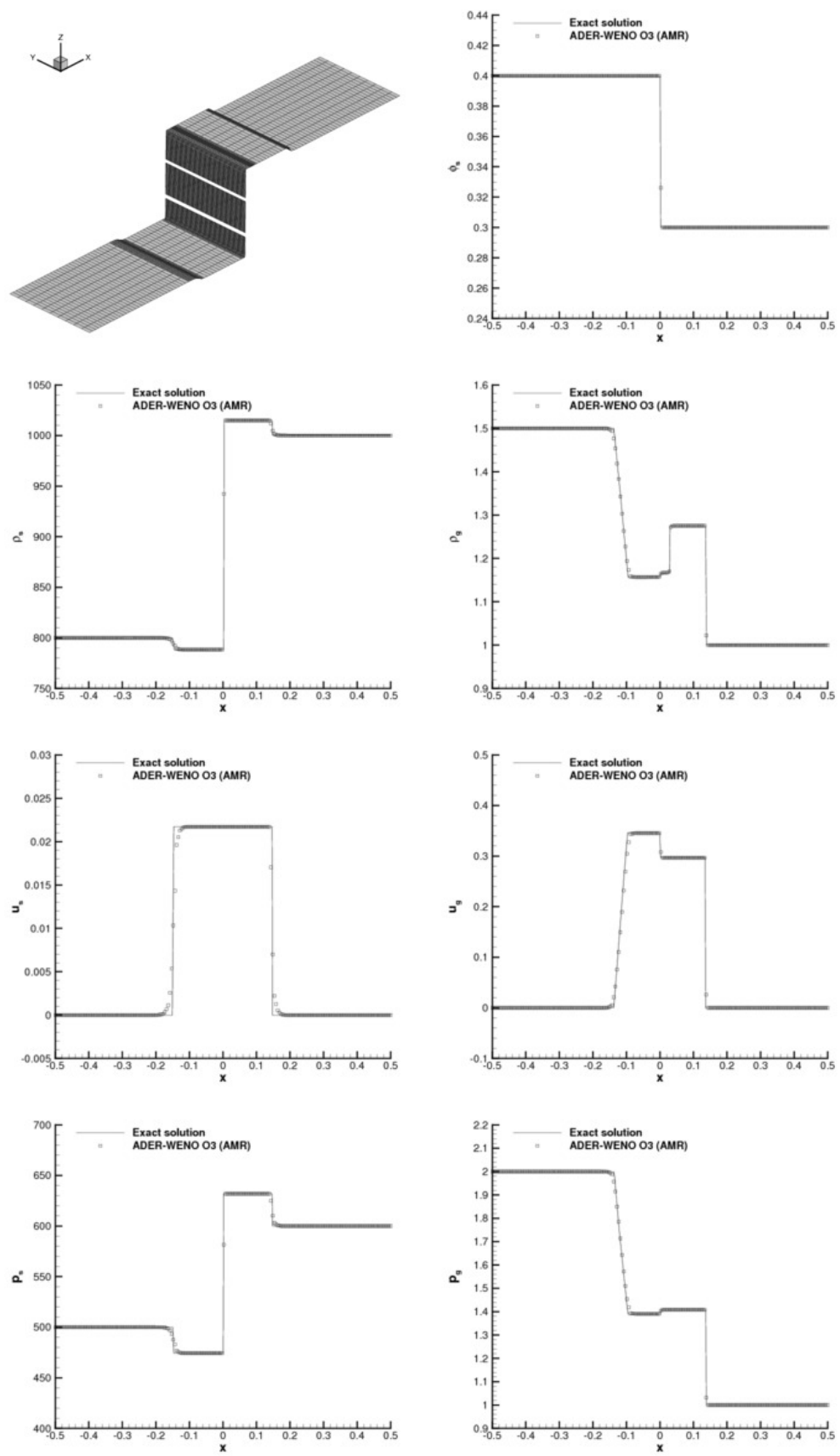

Figure 5: Results for Riemann problem RP2. 

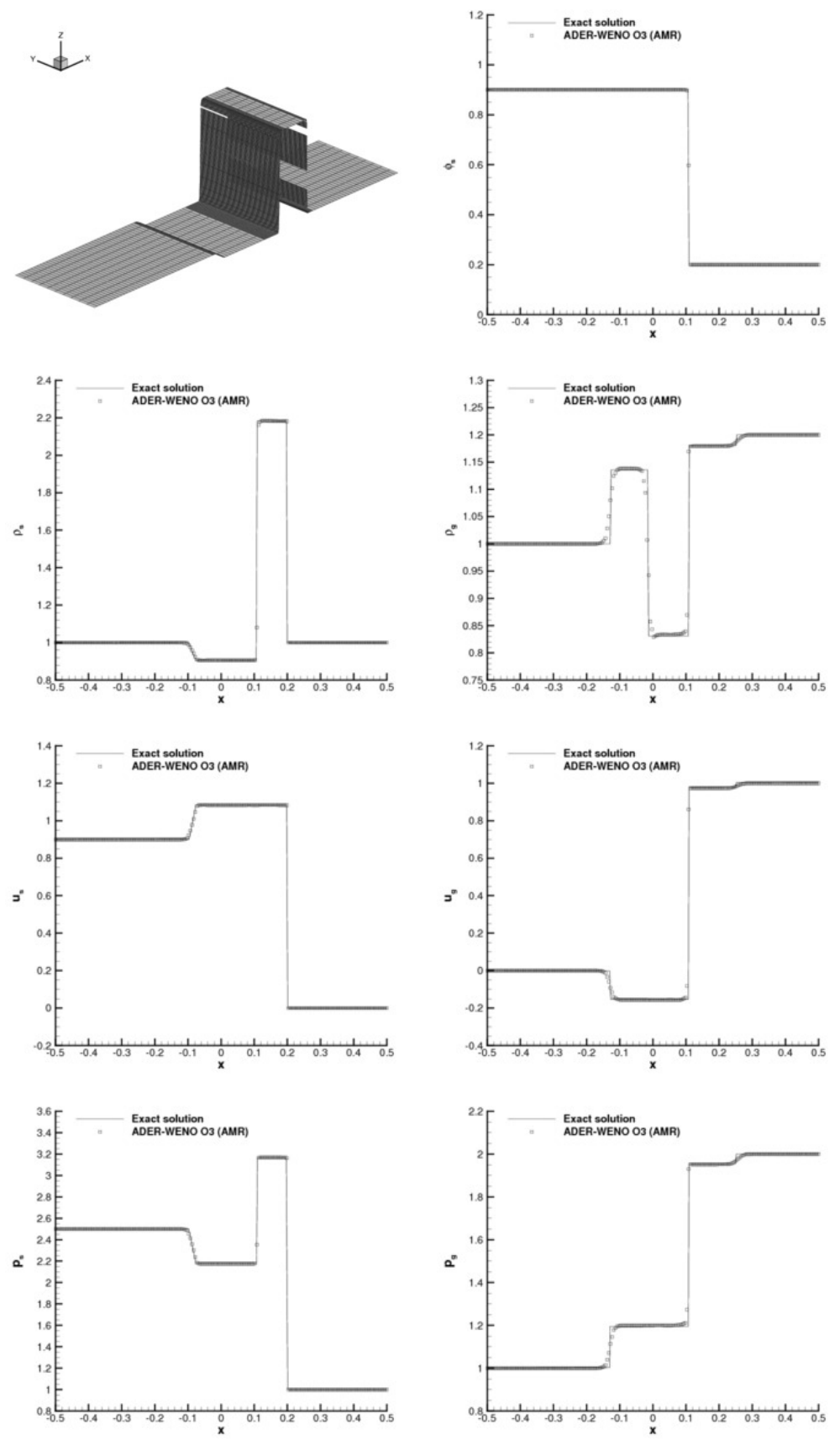

Figure 6: Results for Riemann problem RP3. 

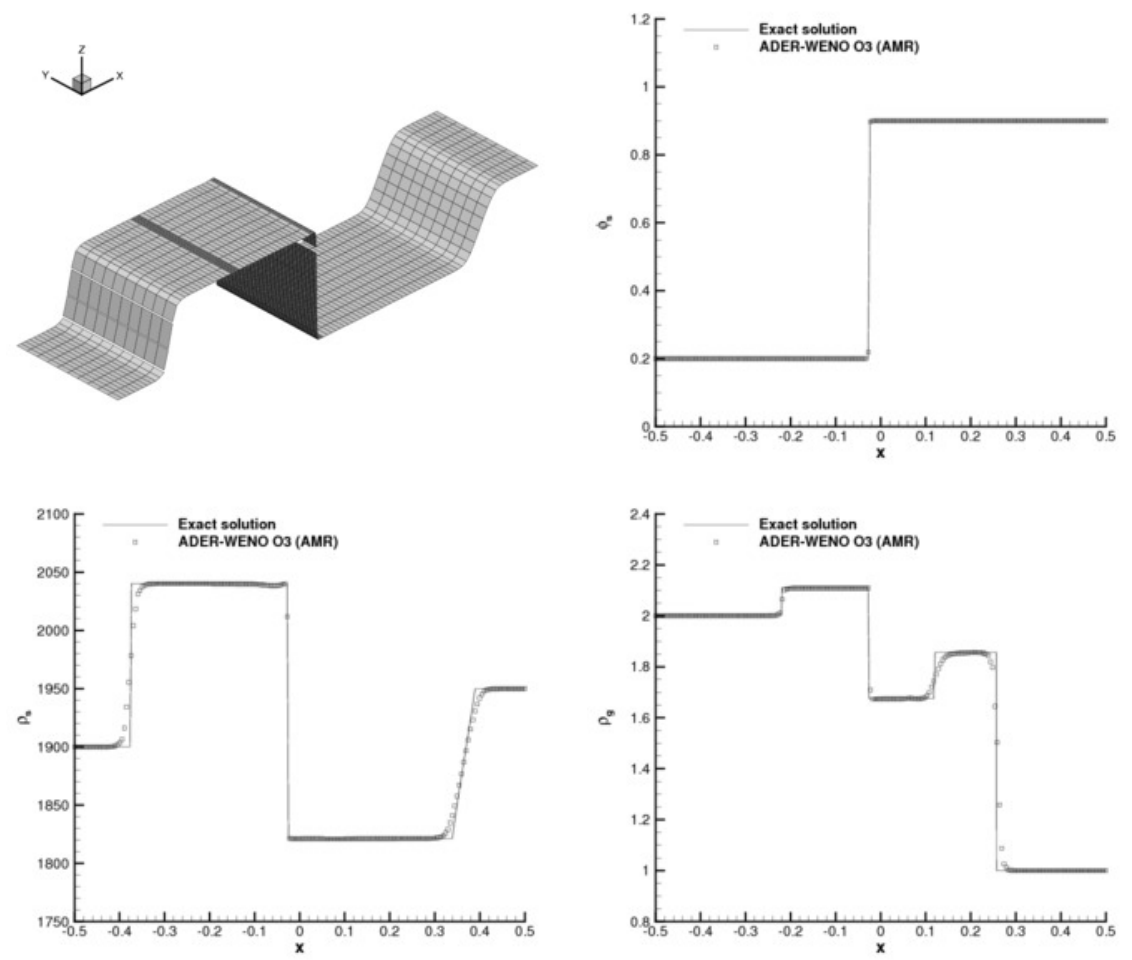

Figure 7: Results for Riemann problem RP4. 

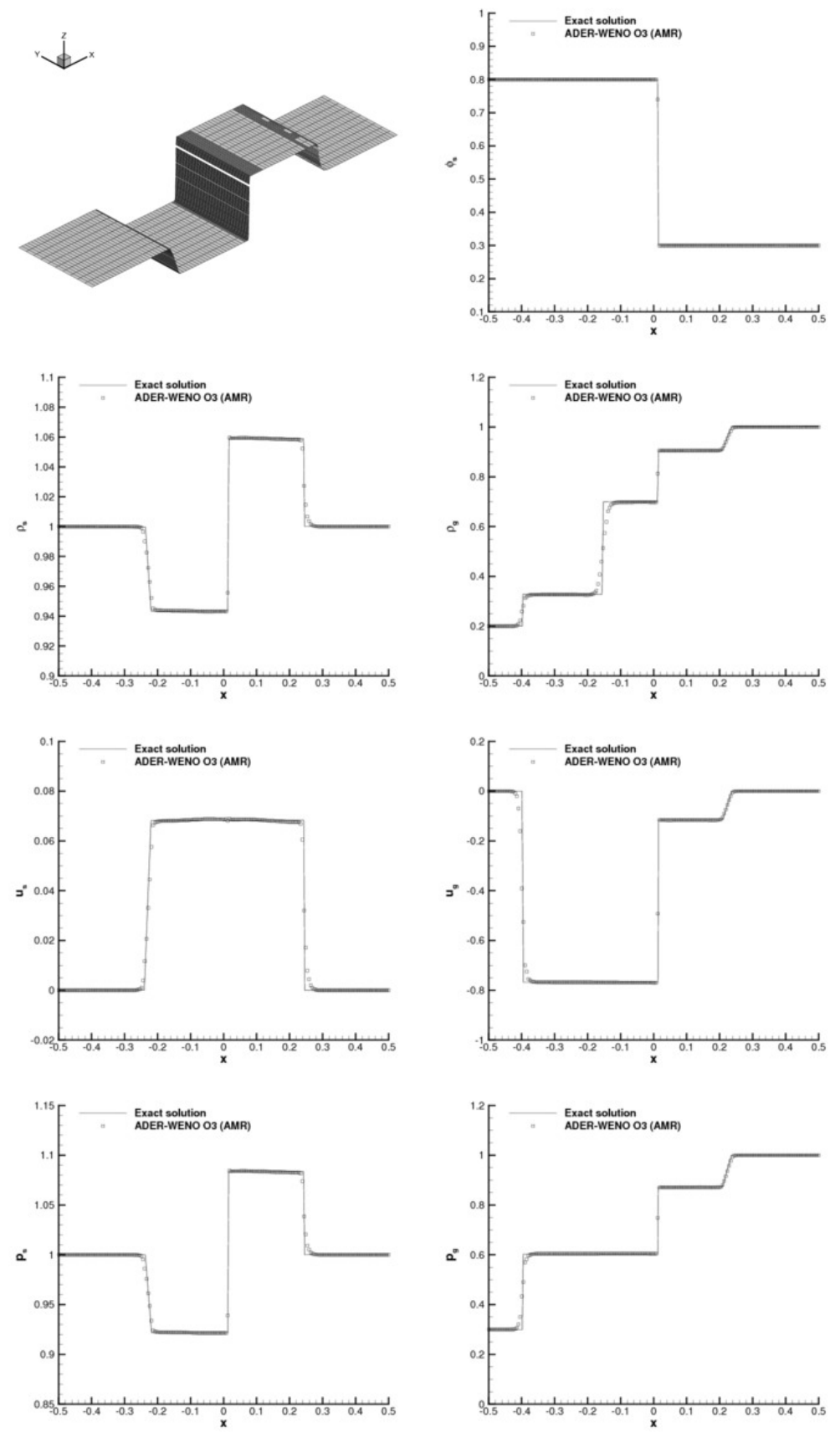

Figure 8: Results for Riemann problem RP5. 

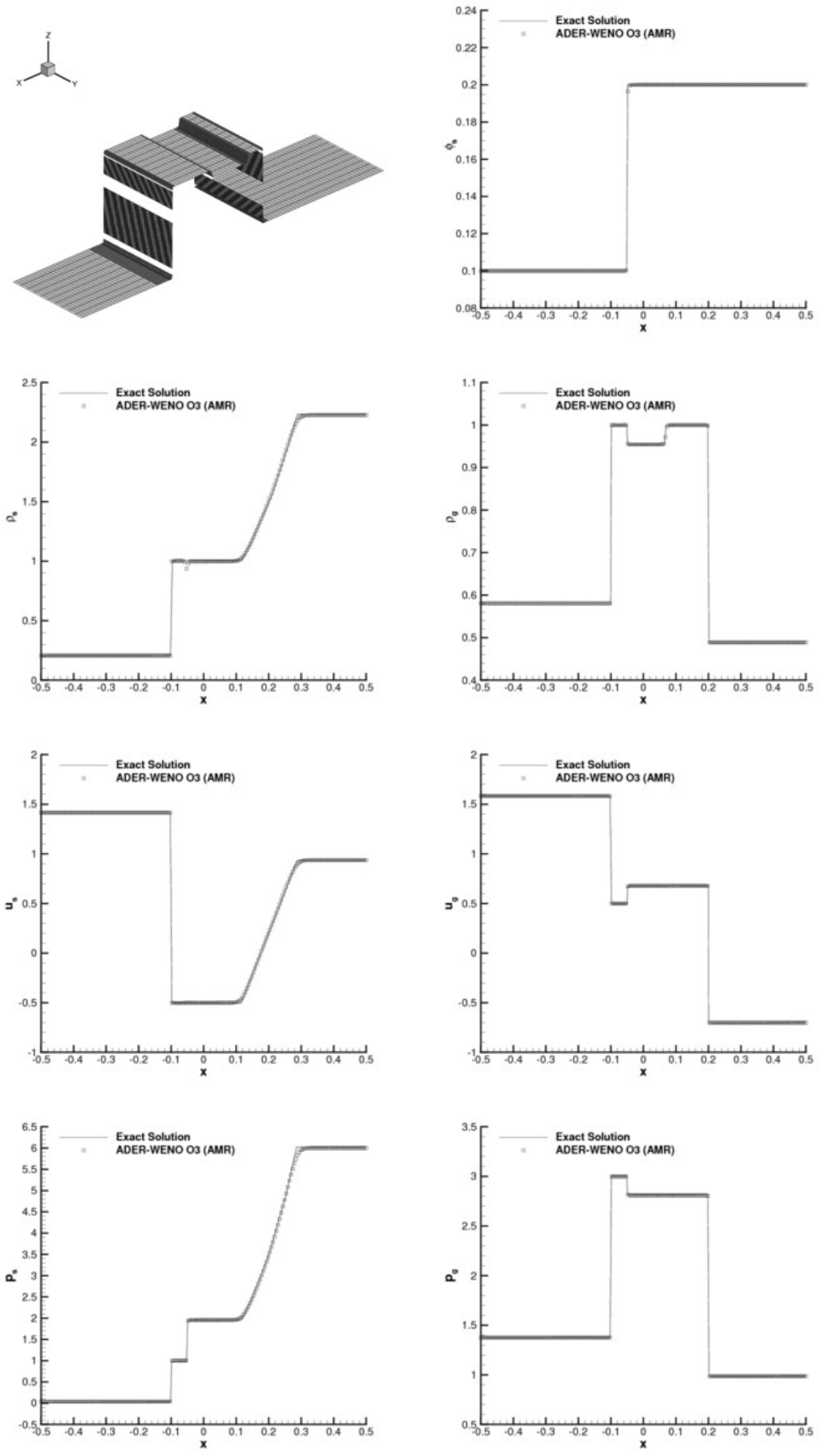

Figure 9: Results for Riemann problem RP6. 
inner and outer states $\mathbf{u}_{i}$ and $\mathbf{u}_{o}$ for the two test cases that we have considered are reported in detail in Table 3. Analogously to the compressible Euler equations, a reference solution can be obtained after solving an equivalent one-dimensional problem in cylindrical coordinates for the two-dimensional explosion, and in spherical coordinates for the three-dimensional one. This essentially implies that the equivalent one-dimensional problem contains additional algebraic source terms on the right hand side of the equations, which account for the use of curvilinear coordinates, see [88, 36]. The 1D reference solution has been computed using a classical second order TVD method with the Osher Riemann solver [39, 40], using 5000 grid cells. For EPa the parameters for the refinement criterion are $\rho_{1,0}=\rho_{2,0}=1$ and for $\mathrm{EPb}$ we use $\rho_{1,0}=1000$ and $\rho_{2,0}=1$.

Table 2: Initial states left $(\mathrm{L})$ and right $(\mathrm{R})$ for the Riemann problems solved in 2D. Values for $\gamma_{i}, \pi_{i}$ and the final time $t_{e}$ are also given.

\begin{tabular}{|c|c|c|c|c|c|c|c|c|}
\hline & $\rho_{s}$ & $u_{s}$ & $p_{s}$ & $\rho_{g}$ & $u_{g}$ & $p_{g}$ & $\phi_{s}$ & $t_{e}$ \\
\hline RP1 [29]: & & $\gamma_{s}=$ & $1.4, \pi$ & $=0$ & $\gamma_{g}=1.4$ & $\pi_{g}=0$ & & \\
\hline $\mathrm{L}$ & 1.0 & 0.0 & 1.0 & 0.5 & 0.0 & 1.0 & 0.4 & 0.10 \\
\hline $\mathrm{R}$ & 2.0 & 0.0 & 2.0 & 1.5 & 0.0 & 2.0 & 0.8 & \\
\hline RP2 [29]: & & $\gamma_{s}=$ & $.0, \quad \pi_{s}$ & $=100$, & $\gamma_{g}=1.4$ & $\pi_{g}=0$ & & \\
\hline $\mathrm{L}$ & 800.0 & 0.0 & 500.0 & 1.5 & 0.0 & 2.0 & 0.4 & 0.10 \\
\hline $\mathrm{R}$ & 1000.0 & 0.0 & 600.0 & 1.0 & 0.0 & 1.0 & 0.3 & \\
\hline RP3 [29]: & & $\gamma_{s}=$ & $1.4, \pi$ & $=0$ & $\gamma_{g}=1.4$ & $\pi_{g}=0$ & & \\
\hline $\mathrm{L}$ & 1.0 & 0.9 & 2.5 & 1.0 & 0.0 & 1.0 & 0.9 & 0.10 \\
\hline $\mathrm{R}$ & 1.0 & 0.0 & 1.0 & 1.2 & 1.0 & 2.0 & 0.2 & \\
\hline RP4 [80]: & & $\gamma_{s}=3$ & $\pi_{s}$ & 3400 & $\gamma_{g}=1.35$ & $\pi_{g}=$ & & \\
\hline $\mathrm{L}$ & 1900.0 & 0.0 & 10.0 & 2.0 & 0.0 & 3.0 & 0.2 & 0.15 \\
\hline $\mathrm{R}$ & 1950.0 & 0.0 & 1000.0 & 1.0 & 0.0 & 1.0 & 0.9 & \\
\hline RP5 [80]: & & $\gamma_{s}=$ & $1.4, \pi$ & $=0$ & $\gamma_{g}=1.4$ & $\pi_{g}=0$ & & \\
\hline $\mathrm{L}$ & 1.0 & 0.0 & 1.0 & 0.2 & 0.0 & 0.3 & 0.8 & 0.20 \\
\hline $\mathrm{R}$ & 1.0 & 0.0 & 1.0 & 1.0 & 0.0 & 1.0 & 0.3 & \\
\hline RP6 [6]: & & $\gamma_{s}=$ & $1.4, \pi$ & $=0$ & $\gamma_{g}=1.4$ & $\pi_{g}=0$ & & \\
\hline $\mathrm{L}$ & 0.2068 & 1.4166 & 0.0416 & 0.5806 & 1.5833 & 1.375 & 0.1 & 0.10 \\
\hline $\mathrm{R}$ & 2.2263 & 0.9366 & 6.0 & 0.4890 & -0.70138 & 0.986 & 0.2 & \\
\hline
\end{tabular}




\subsubsection{D computations}

We have first evolved the two models $\mathrm{EPa}$ and $\mathrm{EPb}$ in two spatial dimensions, by adopting a fourth order ADER-WENO-AMR scheme with two levels of refinement over a computational domain given by $[-1 ; 1] \times[1 ; 1]$. The level zero grid is composed by $50 \times 50$ cells, which are immediately refined according to the refinement criterion applied to the initial conditions. A representative example of the grid at time $t=0$ and at the final time $t=0.2$ is shown in Fig. 10 for the model EPb. The final grid (right panel) is composed by 50020 cells. Fig. 11 and Fig. 12 report the results of the computation for the two models $\mathrm{EPa}$ and $\mathrm{EPb}$ by comparing them with the reference solution.

\subsubsection{D computations}

We have then evolved the same models EPa and EPb in three spatial dimensions, by adopting a third order ADER-WENO-AMR scheme with two levels of refinement over a computational domain given by $[-1 ; 1] \times[1 ; 1] \times$ $[-1 ; 1]$. The level zero grid contains $34 \times 34 \times 34$ cells. The final grid at time $t=0.15$, shown as a representative example in Fig. 13 for the model $\mathrm{EPb}$, is composed by 3, 833, 016 cells. Fig. 14 and Fig. 15 , on the other hand, report the solution at time $t=0.15$, compared to the reference one. All the relevant features and waves of the solution are successfully resolved by the scheme, which remains essentially non-oscillatory and performs the correct grid refinement where this is needed.

Table 3: Inner and outer initial states for the two multidimensional explosion test problems.

\begin{tabular}{cccccccc|cccc}
\hline $\mathrm{EPa}$ & $\rho_{s}$ & $p_{s}$ & $u_{s}$ & $\rho_{g}$ & $p_{g}$ & $u_{g}$ & $\phi_{s}$ & $\gamma_{s}$ & $\pi_{s}$ & $\gamma_{g}$ & $\pi_{g}$ \\
\hline Inner & 1. & 1. & 0. & 0.5 & 1.0 & 0. & 0.4 & 1.4 & 0. & 1.4 & 0. \\
Outer & 2. & 2. & 0. & 1.5 & 2.0 & 0. & 0.8 & & & & \\
\hline \hline $\mathrm{EPb}$ & $\rho_{s}$ & $p_{s}$ & $u_{s}$ & $\rho_{g}$ & $p_{g}$ & $u_{g}$ & $\phi_{s}$ & $\gamma_{s}$ & $\pi_{s}$ & $\gamma_{g}$ & $\pi_{g}$ \\
\hline Inner & 800. & 500. & 0. & 1.5 & 2.0 & 0. & 0.4 & 3.0 & 100. & 1.4 & 0. \\
Outer & 1000. & 600. & 0. & 1.0 & 1.0 & 0. & 0.3 & & & & \\
\hline
\end{tabular}



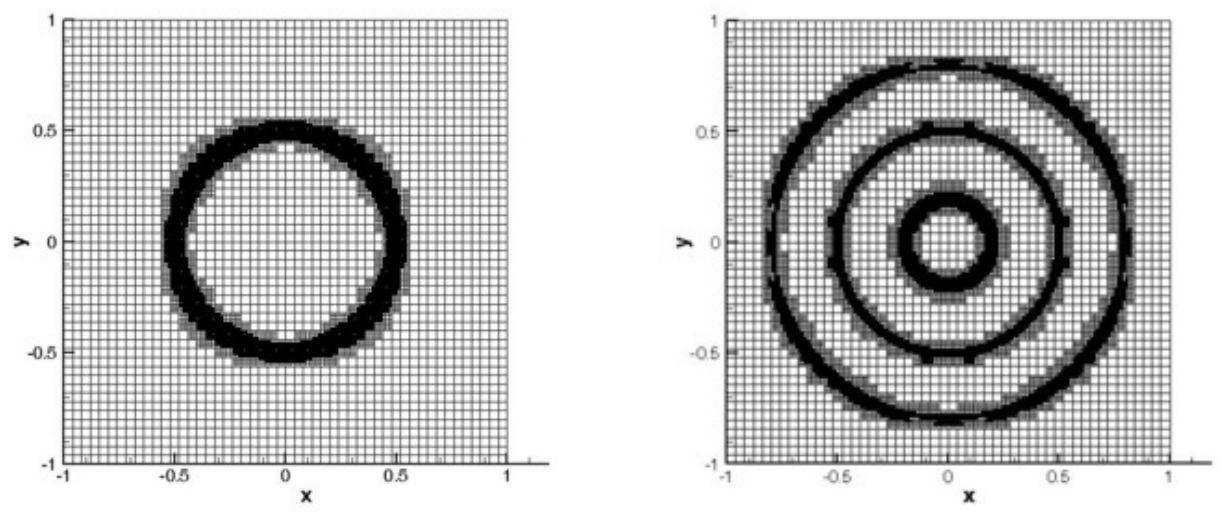

Figure 10: AMR grid structure of the explosion test $\mathrm{EPb}$ in two space dimensions, at time $t=0$ (left panel) and at the final time $t=0.2$ (right panel). Two levels of refinement have been adopted $\left(\ell_{\max }=2\right)$.

\subsection{Shock-bubble interaction}

In this section a strongly simplified shock-bubble interaction-type problem is considered. The initial condition is given by a Riemann problem that leads to a strong isolated shock wave travelling with a shock speed of $s=100$. The pressure jumps over six orders of magnitude across the shock. The initial discontinuity is located at $x=0$ and the left and right initial states, which are connected by a Hugoniot curve, are summarized in Table 4. A bubble of radius $R=0.25$ is located at $x=0.5, y=0$. The state inside the bubble is also given in Table 4 . The parameters for the equation of state of each phase are $\gamma_{1}=3.0, \pi_{1}=100, \gamma_{2}=1.4$ and $\pi_{2}=0$. The problem is solved until time $t=0.0025$ in a computational domain $\Omega=[-0.5 ; 3.0] \times[-0.75 ; 0.75]$ that is discretized on the level $\ell=0$ grid using $70 \times 30$ grid zones. Two levels of refinement are used $\left(\ell_{\max }=2\right)$ with a refinement factor of $\mathfrak{r}=4$, which corresponds to a uniform fine grid resolution of $1120 \times 480$. Periodic boundary conditions are employed in $y$ direction and Dirichlet boundary conditions corresponding to the left and right state are imposed in $x$ direction. We choose $\rho_{1,0}=1000$ and $\rho_{2,0}=1$ for the indicator function $\Phi$. The evolution of the liquid density $\rho_{1}$ and the liquid volume fraction $\phi_{1}$ are depicted for various times in Fig. 16. One can observe how the bubble is compressed and accelerated by the incident shock wave and how Richtmyer-Meshkov- 

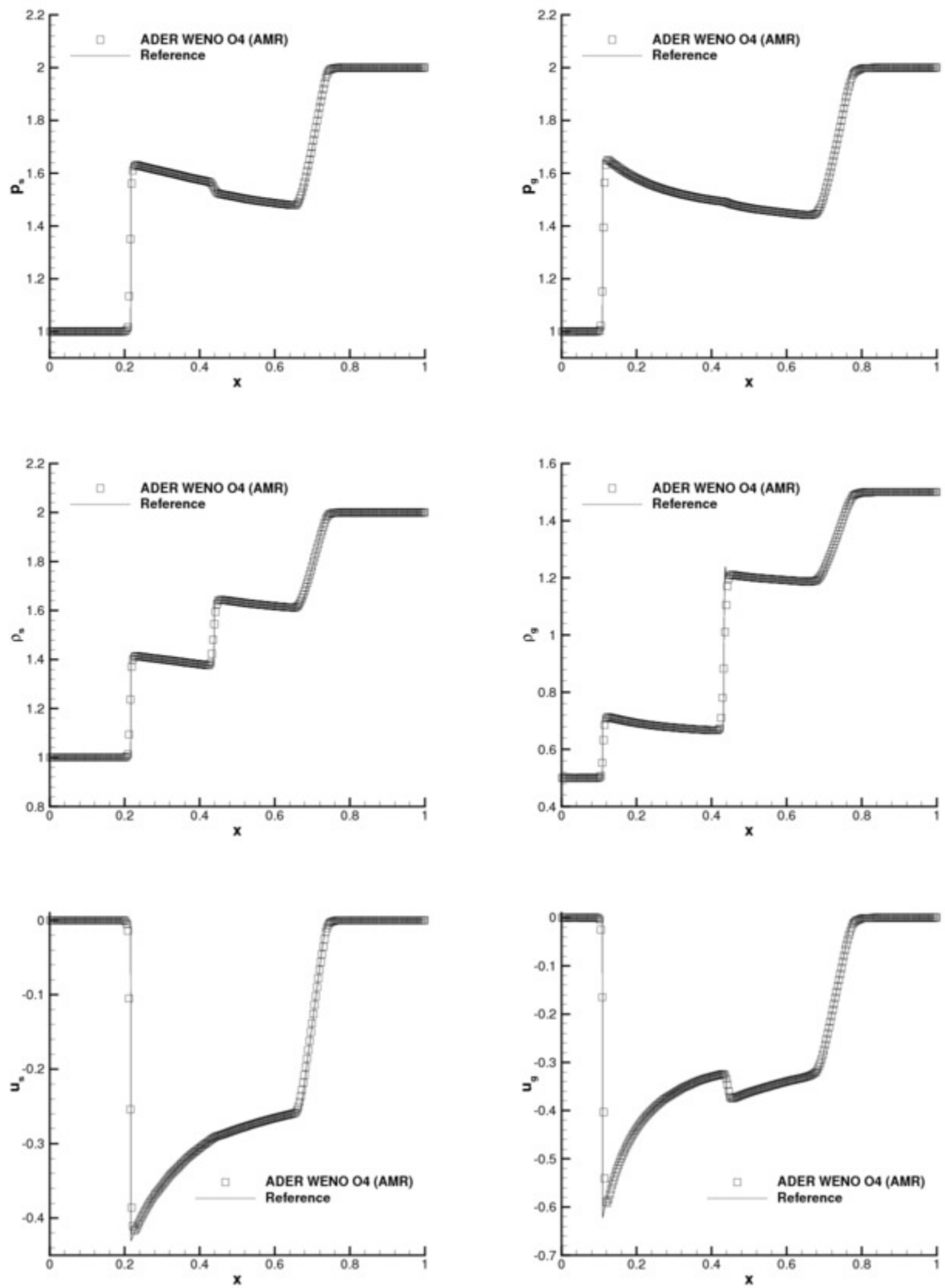

Figure 11: Results of the 2D explosion problem EPa at time $t=0.2$. A cut of various quantities along the $x$-axis is reported, both for the solid (left panels) and for the gas phase (right panels). The 1D reference solution is also shown for comparison. Two levels of refinement have been adopted $\left(\ell_{\max }=2\right)$. 

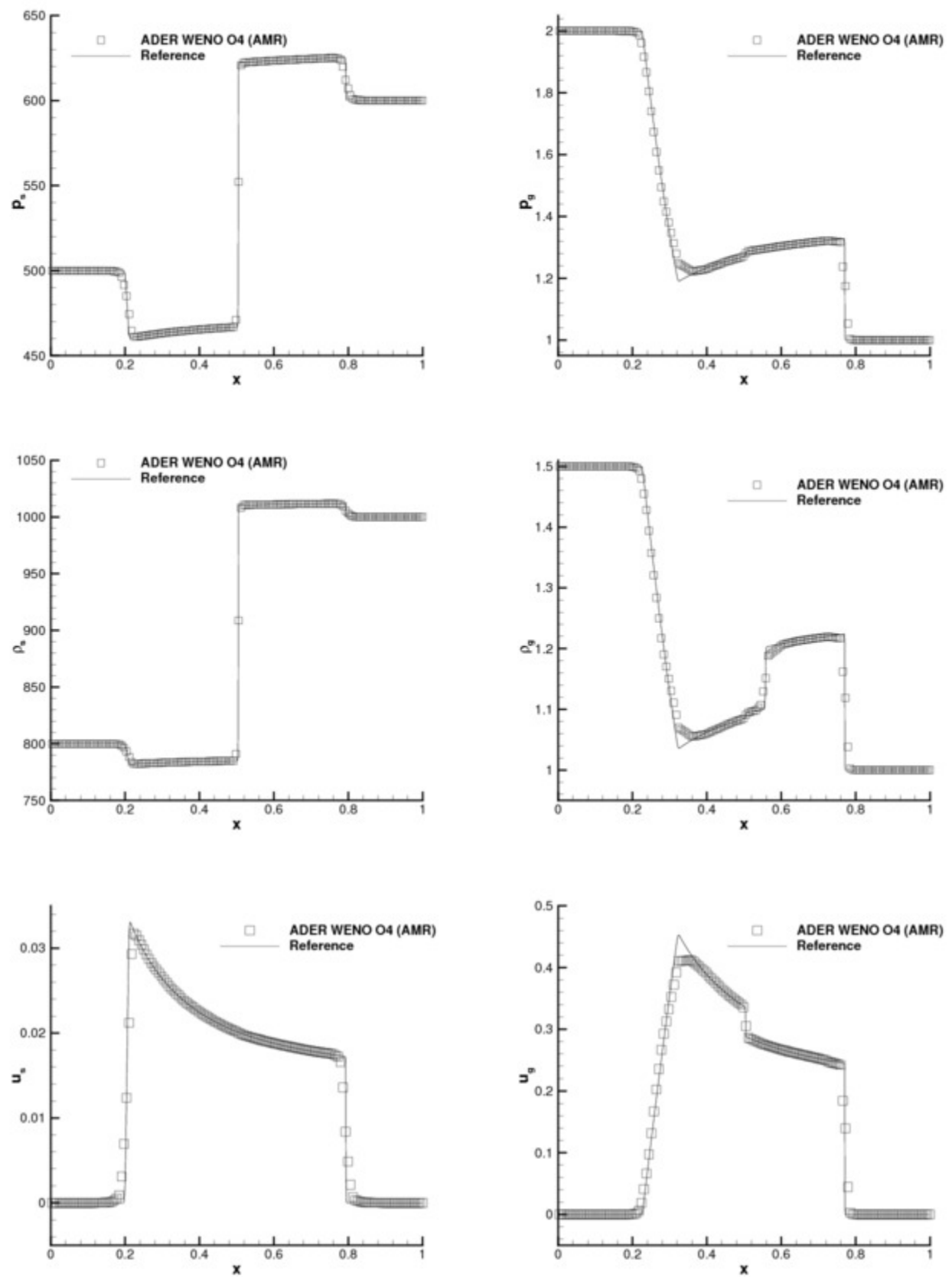

Figure 12: Results of the 2D explosion problem $\mathrm{EPb}$ at time $t=0.2$. A cut of various quantities along the $x$-axis is reported, both for the solid (left panels) and for the gas phase (right panels). The 1D reference solution is also shown for comparison. Two levels of refinement have been adopted $\left(\ell_{\max }=2\right)$. 


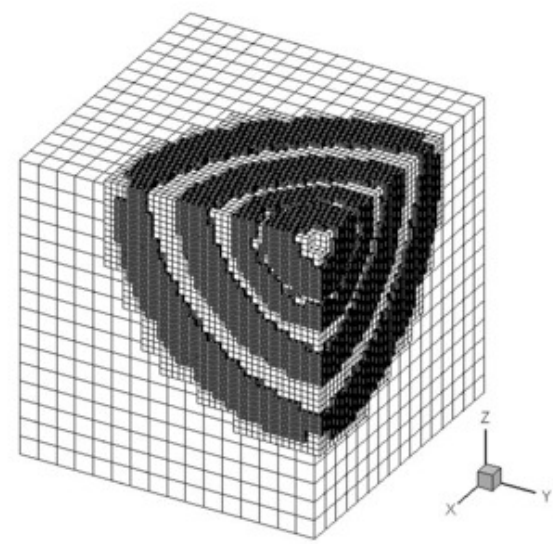

Figure 13: AMR grid structure of the explosion test $\mathrm{EPb}$ in three space dimensions. Two levels of refinement have been adopted $\left(\ell_{\max }=2\right)$, starting from a uniform $34 \times 34 \times 34$ grid.

type flow instabilities occur at the bubble border at later times. One further notes the shock wave reflected by the bubble. A zoom into the AMR grid at the final time $t=0.0025$ is depicted in Fig. 17. The results of this section are only considered as qualitative, in order to test the robustness of our high order AMR method in the presence of strong shock waves in liquids. Here, the high order ADER-WENO AMR scheme has been applied to the full seven equation Baer-Nunziato model, but without taking into account any interphase drag and pressure relaxation, hence no comparison with experimental data can be made for our results. A very detailed quantitative study of shock-bubble interactions with comparison against experimental data has been carried out in [77] using second order accurate high resolution shock capturing schemes together with AMR.

Table 4: Left (L), right (R) and bubble (B) state for the shock-bubble interaction problem.

\begin{tabular}{lccccccc}
\hline & $\rho_{1}$ & $u_{1}$ & $p_{1}$ & $\rho_{2}$ & $u_{2}$ & $p_{2}$ & $\phi_{1}$ \\
\hline Left (L) & 1999.939402 & 49.998485 & 4999849.5 & 1.0 & 0.0 & 1.0 & 0.75 \\
Right (R) & 1000.0 & 0.0 & 1.0 & 1.0 & 0.0 & 1.0 & 0.75 \\
Bubble (B) & 1000.0 & 0.0 & 1.0 & 1.0 & 0.0 & 1.0 & 0.25 \\
\hline
\end{tabular}



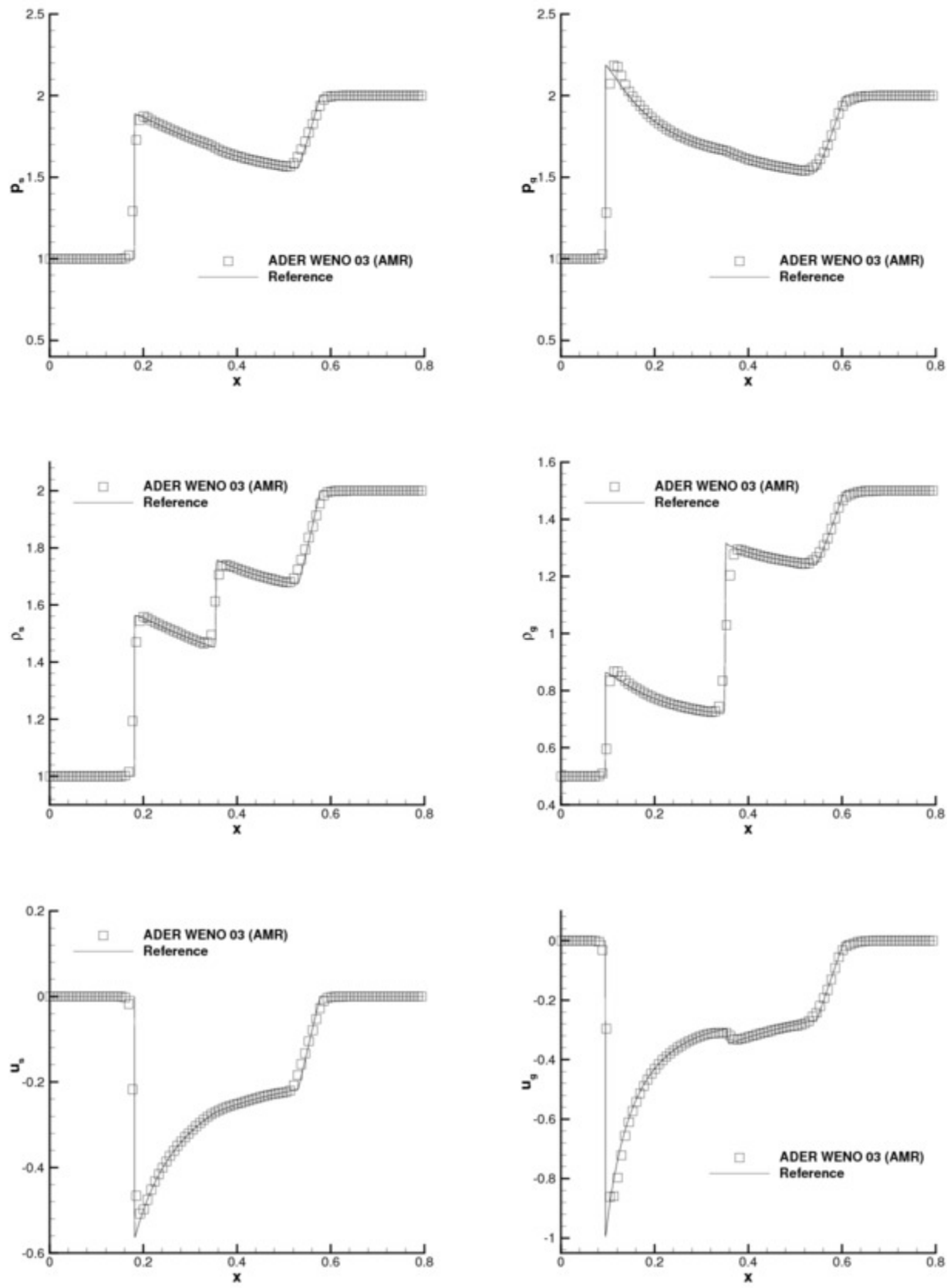

Figure 14: Results of the 3D explosion problem EPa at time $t=0.15$. A cut of various quantities along the $x$-axis is reported, both for the solid (left panels) and for the gas phase (right panels). The 1D reference solution is also shown for comparison. Two levels of refinement have been adopted $\left(\ell_{\max }=2\right)$, starting from a uniform $34 \times 34 \times 34$ grid. 

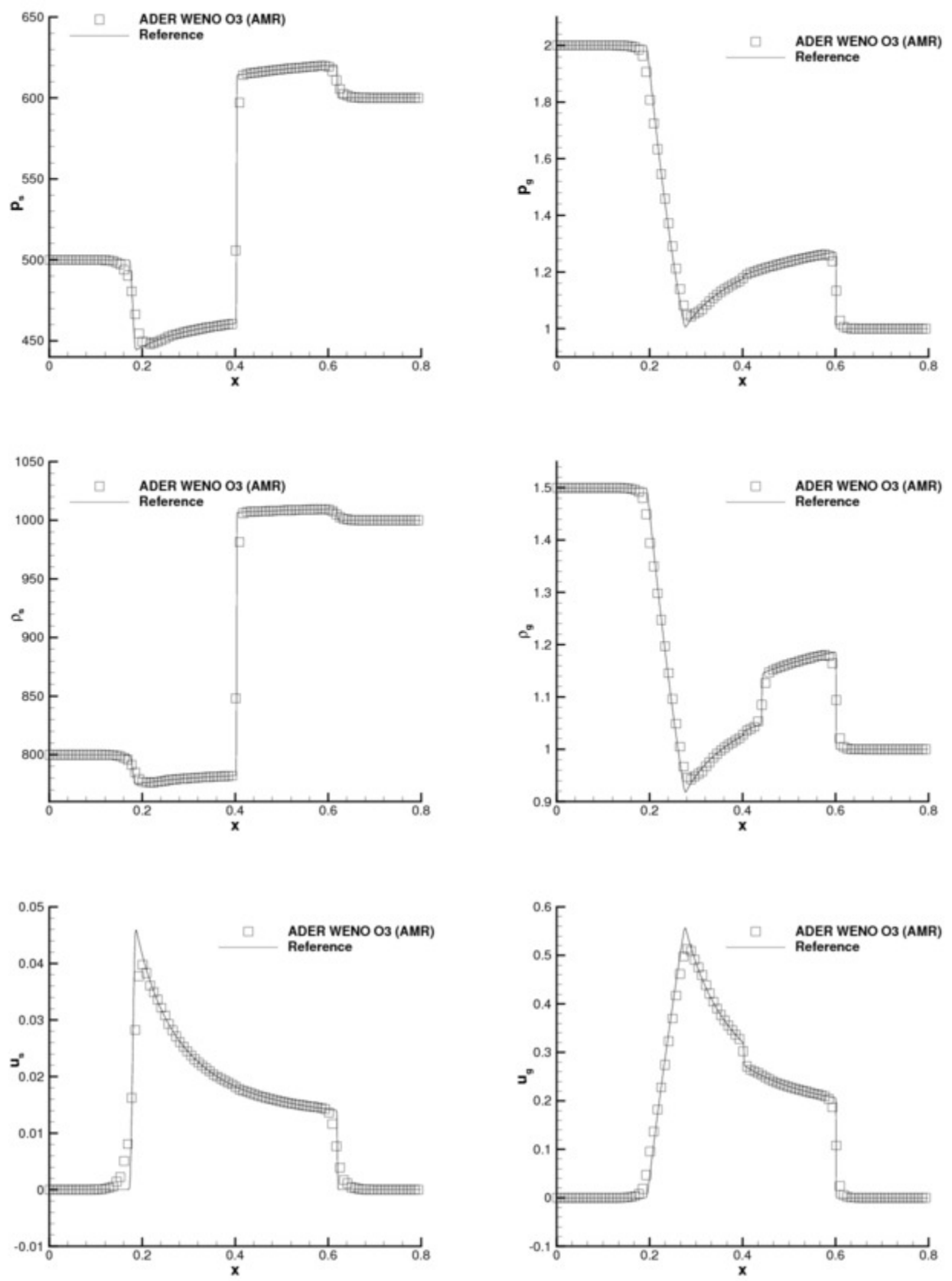

Figure 15: Results of the 3D explosion problem $\mathrm{EPb}$ at time $t=0.15$. A cut of various quantities along the $x$-axis is reported, both for the solid (left panels) and for the gas phase (right panels). The 1D reference solution is also shown for comparison. Two levels of refinement have been adopted $\left(\ell_{\max }=2\right)$, starting from a uniform $34 \times 34 \times 34$ grid. 

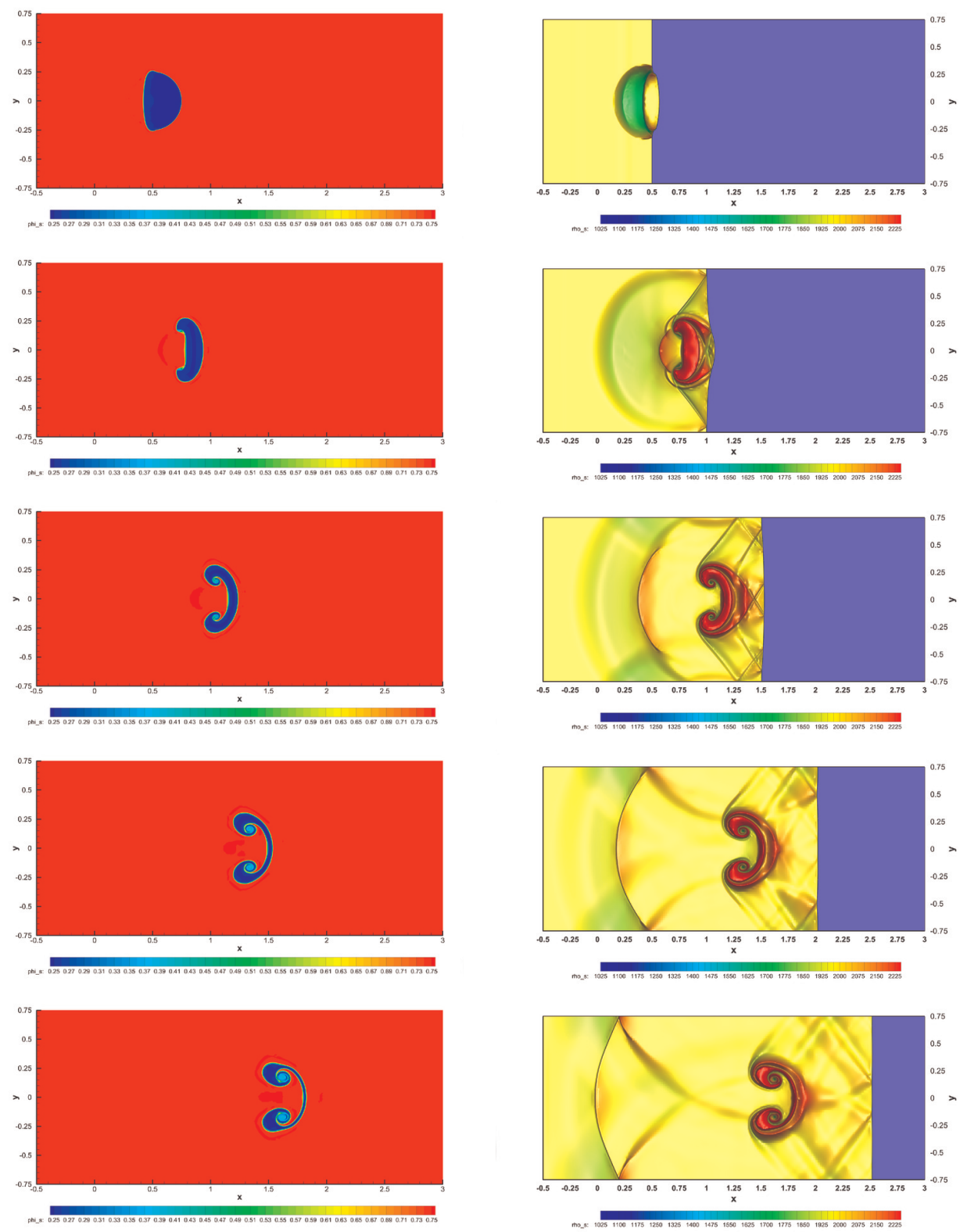

Figure 16: Liquid volume fraction (left) and liquid density (right) of the shock-bubble interaction problem at times $t=0.005, t=0.010, t=0.015, t=0.020$ and $t=0.025$ (from top to bottom) using a third order ADER-WENO scheme with AMR. 


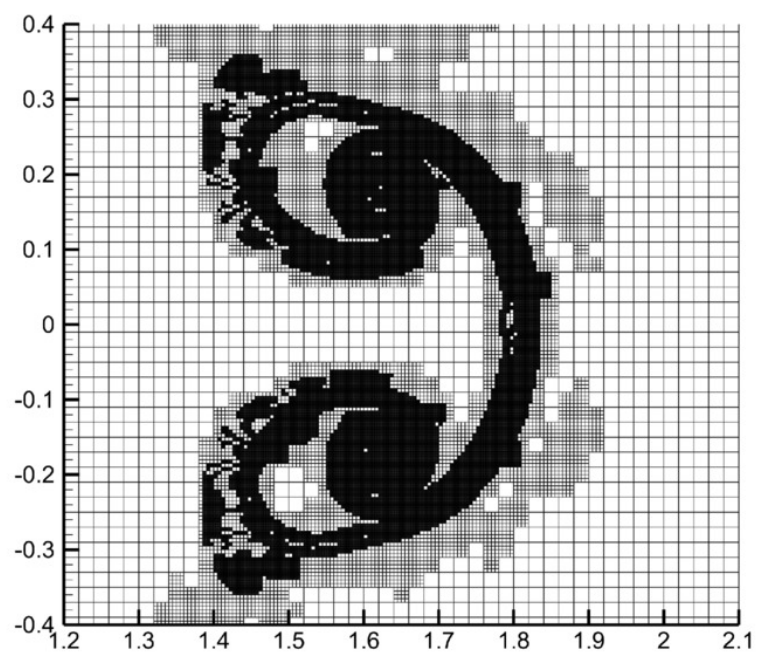

Figure 17: Zoom into the AMR grid at time $t=0.025$ for the shock-bubble interaction problem. 


\subsection{An application to complex free surface flows}

A reduced version of the Baer-Nunziato model (40) can also be used for the simulation of complex non-hydrostatic free surface flows, as proposed in [31, 32]. The reduced three-equation version of the PDE system (40) has been obtained in [31, 32] after introducing the following simplifying assumptions: i) all pressures are relative with respect to the atmospheric reference pressure, which is assumed to be constant and zero everywhere and for all times; ii) the influence of the gas phase onto the liquid can be neglected, hence the evolution equations for the gas phase can be dropped in (40); iii) the pressure of the liquid phase is computed by the Tait equation of state [12, which is a barotropic EOS and therefore also allows the energy equation of the liquid to be removed from the governing PDE system. The Tait EOS reads

$$
p_{1}=k_{0}\left[\left(\frac{\rho_{1}}{\rho_{0}}\right)^{\gamma}-1\right],
$$

where $\rho_{1}, \rho_{0}$ are the liquid density and the reference liquid density at standard conditions, respectively. The compressibility of the fluid is governed by the constants $k_{0}$ and $\gamma$. To avoid low Mach number problems, an artificial Mach number of the order $M \in[0.1 ; 0.3]$ is chosen for typical environmental free surface problems. If real compressibility effects play a role, such as in industrial high pressure applications, the proper values $k_{0}=3.2 \cdot 10^{8} \mathrm{~Pa}$, $\rho_{0}=1000 \mathrm{~kg} / \mathrm{m}^{3}$ and $\gamma=7$ for real water can be chosen, which lead to the real sound speed in water of approximately $c=1500 \mathrm{~m} / \mathrm{s}$.

Introducing the above-mentioned simplifications into system (40) yields the following reduced three-equation model [31, 32]:

$$
\begin{aligned}
& \frac{\partial}{\partial t}(\phi \rho)+\nabla \cdot(\phi \rho \mathbf{v})=0 \\
& \frac{\partial}{\partial t}(\phi \rho \mathbf{v})+\nabla \cdot(\phi(\rho \mathbf{v} \mathbf{v}+p \mathbf{I}))=\phi \rho \mathbf{g}, \\
& \frac{\partial}{\partial t} \phi+\mathbf{v} \cdot \nabla \phi=0 .
\end{aligned}
$$

The subscript 1 has been dropped to ease notation. The mass and momentum equations are fully conservative in the system above since the interface pressure $p_{I}=p_{2}=0$, while the advection equation for the volume fraction remains non-conservative. In (53) the state vector is $\mathbf{Q}=(\phi \rho, \phi \rho \mathbf{v}, \phi)$ and $\mathbf{g}$ is the gravity vector acting along the vertical direction $y$, i.e. $\mathbf{g}=(0,-g)$ with $g=9.81 \mathrm{~m} / \mathrm{s}^{2}$. Further details and thorough validations of this model can be found in [31, 32]. 
The last test problem of this paper where we apply the high order ADERWENO scheme with adaptive mesh refinement consists of a dambreak with successive wave impact against a vertical wall. In this test problem the free surface is strongly deformed and the impact against the wall also leads to wave breaking. The setup of this test case is taken from [28, 46]. The rectangular computational domain is defined as $\Omega=[0 ; 3.2] \times[0 ; 2.0]$ with reflective wall boundaries on all sides of the domain apart from the top, which is assumed to be transmissive. The initial liquid domain is $\Omega_{l}=$ $[0 ; 1.2] \times[0 ; 0.6]$, where $\phi=0.999$ is set. The initial velocity is zero and the initial density distribution is chosen such as to obtain a hydrostatic pressure distribution inside the liquid, see [31] for details. Outside the liquid domain, we set the pressure to zero and a low but finite value for the liquid volume fraction is chosen $\left(\phi=10^{-3}\right)$. We use $g=9.81$ and $k_{0}=2.0 \cdot 10^{5}$. The level zero grid contains only $60 \times 40$ cells. We use two levels of refinement $\ell_{\max }=2$ and a refinement factor of $\mathfrak{r}=4$. This corresponds to an equivalent resolution on a uniform fine mesh of $960 \times 640$ elements. A third order ADERWENO method is used together with the little dissipative Osher scheme. The contours of the liquid volume fraction $\phi$ are depicted for three output times in Fig. 18. The results of the present high order AMR method are compared with the results on a uniform fine grid. According to Fig. 18 the two solutions match very well. The AMR grid at the final time $t=1.5$ is depicted in Fig. 19 and contains 47710 cells, while the uniform fine grid contains 614400 cells. This means that for the present test problem the use of an AMR technique allows to obtain almost the same results as with a uniform fine grid, but with 12.88 times less elements. Concerning the AMR overhead we also report the average CPU time per element update (EU) in Table 5, which is the total wallclock time divided by the total number of element updates, normalized with respect to the uniform grid. This figure indicates the overhead introduced by the AMR machinery. For the present test problem, using a third order ADER-WENO AMR scheme it is only $19 \%$, which agrees with the data for the AMR overhead published in [42]. One can note how the AMR algorithm refines the grid in the vicinity of the free surface, which is resolved in a very sharp manner. The flow evolution computed with the present method is also in good agreement with the 3D SPH computations performed in [46] and with the results presented in [31, 32. In Fig. 20 we compare the experimental data for the pressure at the wall 94 with the numerical results obtained with the method proposed in this paper. The amplitude and the arrival time of the first pressure peak due 
to the wave impact at the wall is captured correctly.

\section{Conclusions}

In the present paper the first better than second order accurate pathconservative one-step WENO finite volume scheme with adaptive mesh refinement (AMR) has been presented for the solution of non-conservative hyperbolic systems. The method has been applied in particular to the BaerNunziato model of compressible multiphase flows. It has been shown via a numerical convergence study that the proposed numerical approach reaches the designed high order of accuracy in space and time. Furthermore, the accuracy and robustness of the method has been validated on a large set of test problems, for which either exact or other reference solutions were available. It has been clearly shown that the use of AMR can lead to a significant reduction in grid elements and CPU time compared to simulations performed on fine uniform grids. Compared to our previous publications [36, 40], where high order schemes have been employed without AMR, the material interfaces are much better resolved using the present high order AMR technique. This becomes particularly evident in the results obtained for the shock tube problems solved in Section 5.2.

Future applications of the present high order one-step AMR methodology may concern the simulation of bubbles with phase transition [30] and chemically reacting compressible multiphase flows. For the latter it has already been shown before in literature that AMR techniques can be very useful to resolve all the length scales involved in such multi-scale problems [52].

Table 5: Memory and CPU time comparison of the third order ADER-WENO AMR method and ADER-WENO on a uniform fine grid for the dambreak and wave impact problem of Section 5.5. Memory consumption is measured in number of real elements and CPU time is normalized with respect to the wallclock time for the fine uniform mesh. The normalized average time per element update $(\mathrm{EU})$ is given in the last row to quantify the overhead of the AMR framework.

\begin{tabular}{cccc}
\hline & AMR & Uniform & ratio \\
\hline Number of real cells & 47710 & 614400 & 12.877 \\
Total CPU time & 0.1134 & 1.0 & 8.816 \\
Average time per EU & 1.1923 & 1.0 & 0.839 \\
\hline
\end{tabular}



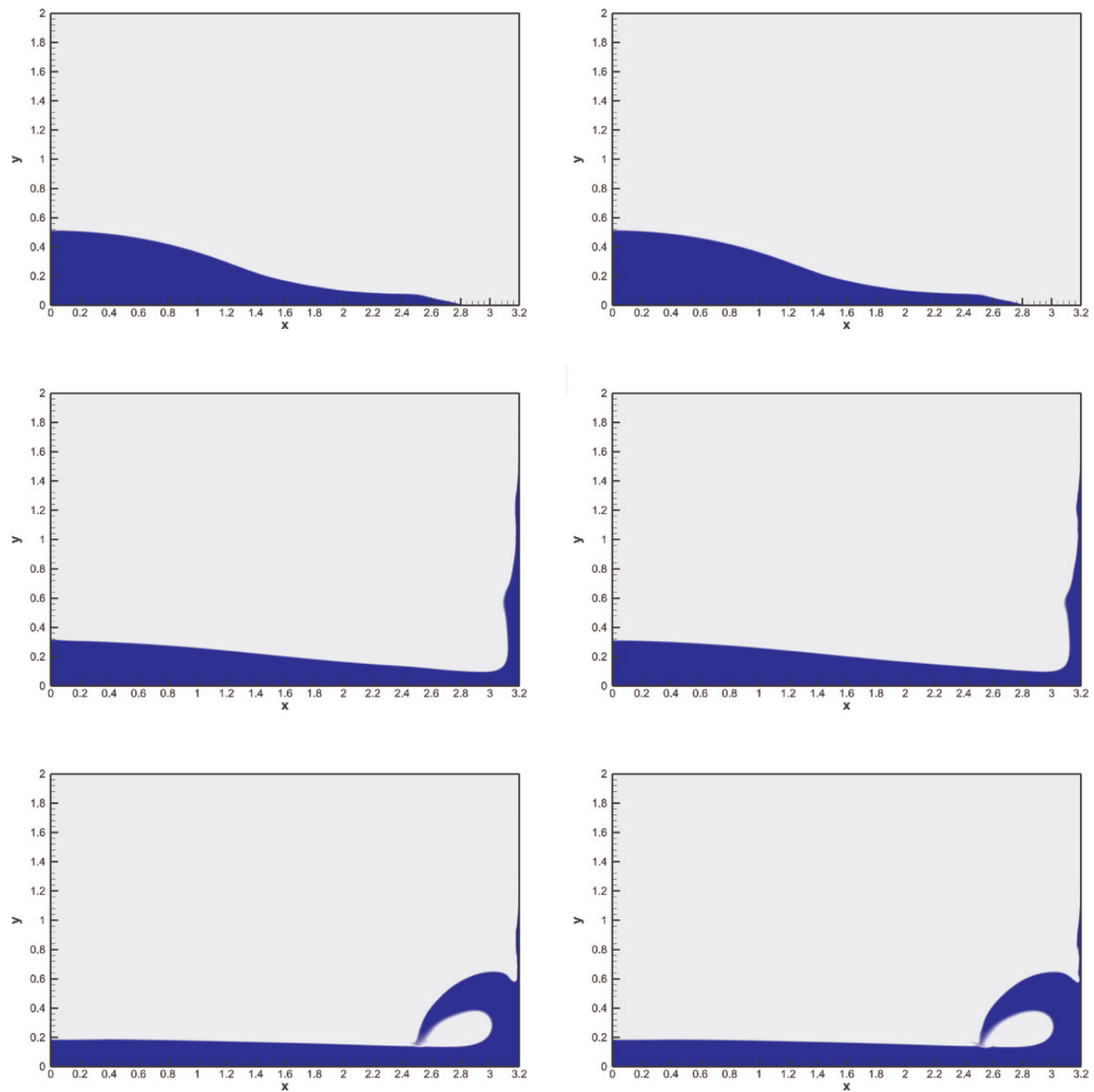

Figure 18: Dambreak and wave-impact problem at times $t=0.5, t=1.0$ and $t=1.5$ using a third order ADER-WENO scheme with AMR (left) and with a uniform fine grid (right). 


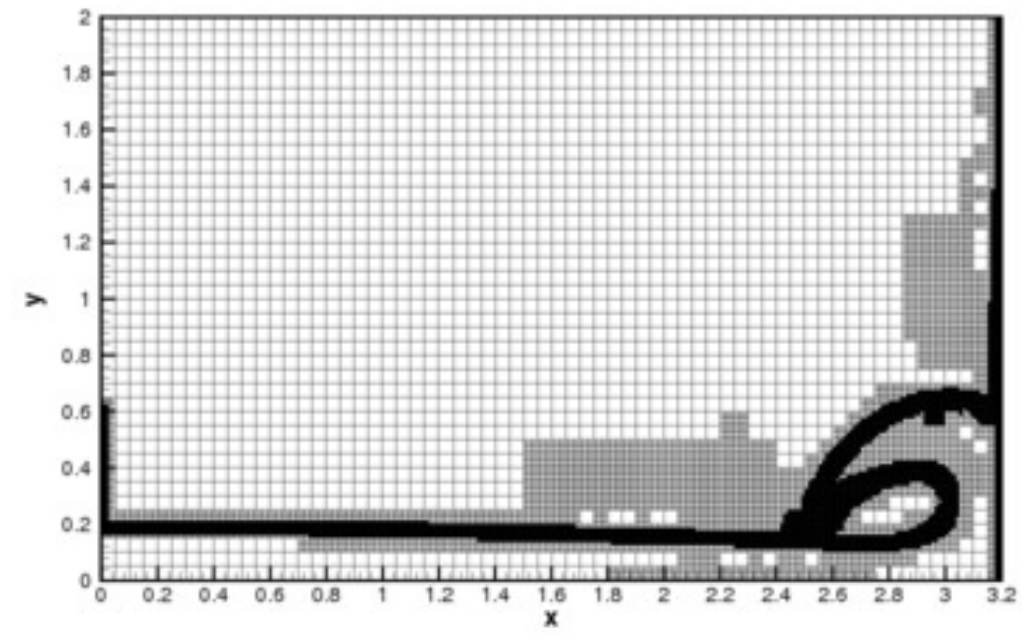

Figure 19: AMR grid for the dambreak and wave impact problem at time $t=1.5$.

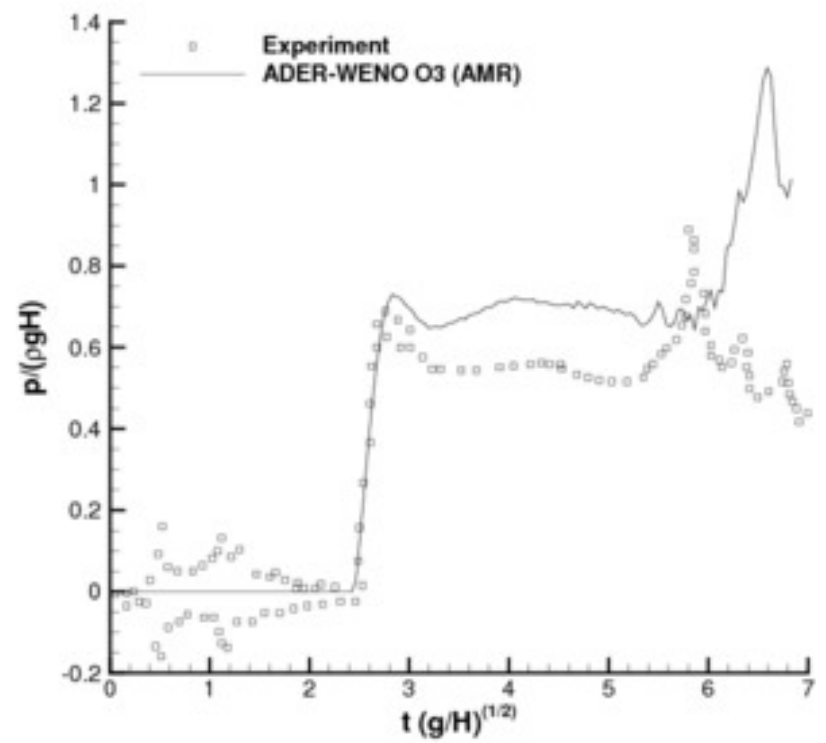

Figure 20: Pressure evolution at the wall. Comparison of experimental data with the computational results. 
Other relevant mathematical models that could be treated in the framework outlined in this paper include the nonconservative debris flow model of Pitman and Le [75] as well as single and multi-layer shallow water equations [24, 73, 25, 3]. In particular for multi-scale tsunami wave simulations the combination of a high order method that allows a coarse grid to discretize the wave propagation in the ocean with a fine grid that allows to follow and resolve all the relevant details on the coastline may be useful.

\section{Acknowledgments}

The research conducted here has been financed by the European Research Council under the European Union's Seventh Framework Programme (FP7/2007-2013) in the frame of the research project STiMulUs, ERC Grant agreement no. 278267. A.H. thanks Fundación Caja Madrid (Spain) for its financial support by a grant under the programme Becas de movilidad para profesores de las universidades públicas de Madrid.

\section{References}

[1] R. Abgrall. How to prevent pressure oscillations in multicomponent flow calculations: A quasi conservative approach. Journal of Computational Physics, 125:150-160, 1996.

[2] R. Abgrall and S. Karni. Computations of Compressible Multifluids. Journal of Computational Physics, 169:594-623, 2001.

[3] R. Abgrall and S. Karni. Two-layer shallow water system: A relaxation approach. SIAM Journal on Scientific Computing, 31:1603-1627, 2009.

[4] R. Abgrall and S. Karni. A comment on the computation of nonconservative products. Journal of Computational Physics, 229:2759$2763,2010$.

[5] R. Abgrall and R. Saurel. Discrete equations for physical and numerical compressible multiphase mixtures. Journal of Computational Physics, 186:361-396, 2003.

[6] N. Andrianov and G. Warnecke. The Riemann problem for the BaerNunziato two-phase flow model. Journal of Computational Physics, 212:434-464, 2004. 
[7] M.R. Baer and J.W. Nunziato. A two-phase mixture theory for the deflagration-to-detonation transition (DDT) in reactive granular materials. J. Multiphase Flow, 12:861-889, 1986.

[8] A. Baeza and P. Mulet. Adaptive mesh refinement techniques for highorder shock capturing schemes for multi-dimensional hydrodynamic simulations. International Journal for Numerical Methods in Fluids, 52:455-471, 2006.

[9] D. S. Balsara, T. Rumpf, M. Dumbser, and C.-D. Munz. Efficient, high accuracy ADER-WENO schemes for hydrodynamics and divergence-free magnetohydrodynamics. Journal of Computational Physics, 228:24802516, April 2009.

[10] D.S. Balsara. Divergence-Free Adaptive Mesh Refinement for Magnetohydrodynamics. Journal of Computational Physics, 174:614-648, 2001.

[11] D.S. Balsara, C. Meyer, M. Dumbser, H. Du, and Z. Xu. Efficient implementation of ADER schemes for Euler and magnetohydrodynamical flows on structured meshes - Speed comparisons with Runge-Kutta methods. Journal of Computational Physics, 235:934-969, 2013.

[12] G.K. Batchelor. An introduction to fluid mechanics. Cambridge University Press, 1974.

[13] J. Bell, M. Berger, J. Saltzman, and M. Welcome. Three-dimensional adaptive mesh refinement for hyperbolic conservation laws. SIAM J. Sci. Comput., 15(1):127-138, January 1994.

[14] M. J. Berger and P. Colella. Local adaptive mesh refinement for shock hydrodynamics. Journal of Computational Physics, 82:64-84, May 1989.

[15] M. J. Berger and A. Jameson. Automatic adaptive grid refinement for the Euler equations. AIAA Journal, 23:561-568, April 1985.

[16] M. J. Berger and R. LeVeque. Adaptive mesh refinement using wavepropagation algorithms for hyperbolic systems. SIAM Journal on $\mathrm{Nu}$ merical Analysis, 35:2298-2316, December 1998.

[17] M. J. Berger and J. Oliger. Adaptive Mesh Refinement for Hyperbolic Partial Differential Equations. Journal of Computational Physics, 53:484, March 1984. 
[18] M. Berndt, J. Breil, S. Galera, M. Kucharik, P.H. Maire, and M. Shashkov. Two-step hybrid conservative remapping for multimaterial arbitrary Lagrangian-Eulerian methods. Journal of Computational Physics, 230:6664-6687, 2011.

[19] J. Breil, S. Galera, and P.H. Maire. Multi-material ALE computation in inertial confinement fusion code CHIC. Computers and Fluids, 46:161167, 2011.

[20] G. Carré, S. Del Pino, B. Després, and E. Labourasse. A cell-centered lagrangian hydrodynamics scheme on general unstructured meshes in arbitrary dimension. Journal of Computational Physics, 228:5160 - 5183, 2009.

[21] J. J. Carroll-Nellenback, B. Shroyer, A. Frank, and C. Ding. Efficient Parallelization for AMR MHD Multiphysics Calculations; Implementation in AstroBEAR. ArXiv e-prints, December 2011.

[22] C.E. Castro, M. Käser, and E.F. Toro. Space-time adaptive numerical methods for geophysical applications. Philosophical Transactions of the Royal Society A: Mathematical, Physical and Engineering Sciences, 367:4613-4631, 2009.

[23] M.J. Castro, J.M. Gallardo, J.A. López, and C. Parés. Well-balanced high order extensions of Godunov's method for semilinear balance laws. SIAM Journal of Numerical Analysis, 46:1012-1039, 2008.

[24] M.J. Castro, J.M. Gallardo, and C. Parés. High-order finite volume schemes based on reconstruction of states for solving hyperbolic systems with nonconservative products. applications to shallow-water systems. Mathematics of Computations, 75:1103-1134, 2006.

[25] M.J. Castro, J.A. García-Rodríguez, J.M. González-Vida, J. Macías, C. Parés, and M.E. Vázquez-Cendón. Numerical simulation of two-layer shallow water flows through channels with irregular geometry. Journal of Computational Physics, 195:202-235, 2004.

[26] M.J. Castro, P.G. LeFloch, M.L. Muñoz-Ruiz, and C. Parés. Why many theories of shock waves are necessary: Convergence error in formally path-consistent schemes. Journal of Computational Physics, 227:81078129, 2008. 
[27] J. Cheng and C.W. Shu. A high order ENO conservative Lagrangian type scheme for the compressible Euler equations. Journal of Computational Physics, 227:1567-1596, 2007.

[28] A. Colagrossi and M. Landrini. Numerical simulation of interfacial flows by Smoothed Particle Hydrodynamics. Journal of Computational Physics, 191:448-475, 2003.

[29] V. Deledicque and M.V. Papalexandris. An exact Riemann solver for compressible two-phase flow models containing non-conservative products. Journal of Computational Physics, 222:217-245, 2007.

[30] W. Dreyer, F. Duderstadt, M. Hantke, and G. Warnecke. Bubbles in liquids with phase transition Part 1. On phase change of a single vapor bubble in liquid water. Continuum Mechanics and Thermodynamics, 24:461-483, 2012.

[31] M. Dumbser. A simple two-phase method for the simulation of complex free surface flows. Computer Methods in Applied Mechanics and Engineering, 200:1204-1219, 2011.

[32] M. Dumbser. A Diffuse Interface Method for Complex ThreeDimensional Free Surface Flows. Computer Methods in Applied Mechanics and Engineering, 257:47-64, 2013.

[33] M. Dumbser, D.S. Balsara, E.F. Toro, and C.D. Munz. A unified framework for the construction of one-step finite-volume and discontinuous Galerkin schemes. Journal of Computational Physics, 227:8209-8253, 2008.

[34] M. Dumbser and W. Boscheri. High-Order Unstructured Lagrangian One-Step WENO Finite Volume Schemes for Non-conservative Hyperbolic Systems: Applications to Compressible Multi-Phase Flows. Computers and Fluids. submitted to. http://arxiv.org/abs/1304.4816.

[35] M. Dumbser, C. Enaux, and E.F. Toro. Finite volume schemes of very high order of accuracy for stiff hyperbolic balance laws. Journal of Computational Physics, 227:3971-4001, 2008. 
[36] M. Dumbser, A. Hidalgo, M. Castro, C. Parés, and E.F. Toro. FORCE schemes on unstructured meshes II: Non-conservative hyperbolic systems. Computer Methods in Applied Mechanics and Engineering, 199:625-647, 2010.

[37] M. Dumbser, M. Käser, V.A Titarev, and E.F. Toro. Quadrature-free non-oscillatory finite volume schemes on unstructured meshes for nonlinear hyperbolic systems. Journal of Computational Physics, 226:204-243, 2007.

[38] M. Dumbser, M. Käser, and E. F. Toro. An arbitrary high order discontinuous Galerkin method for elastic waves on unstructured meshes V: Local time stepping and p-adaptivity. Geophysical Journal International, 171:695-717, 2007.

[39] M. Dumbser and E.F. Toro. On universal Osher-type schemes for general nonlinear hyperbolic conservation laws. Communications in Computational Physics, 10:635-671, 2011.

[40] M. Dumbser and E.F. Toro. A simple extension of the Osher Riemann solver to non-conservative hyperbolic systems. Journal of Scientific Computing, 48:70-88, 2011.

[41] M. Dumbser and O. Zanotti. Very high order PNPM schemes on unstructured meshes for the resistive relativistic MHD equations. Journal of Computational Physics, 228:6991-7006, 2009.

[42] M. Dumbser, O. Zanotti, A. Hidalgo, and D. S. Balsara. ADER-WENO Finite Volume Schemes with Space-Time Adaptive Mesh Refinement. ArXiv e-prints, December 2012.

[43] B. Van Der Holst et al. Crash: A block-adaptive-mesh code for radiative shock hydrodynamics - Implementation and verification. Astrophysical Journal, Supplement Series, 194:23, 2011.

[44] R. Fedkiw, T. Aslam, B. Merriman, and S. Osher. A non-oscillatory Eulerian approach to interfaces in multimaterial flows (the ghost fluid method). Journal of Computational Physics, 152:457-492, 1999. 
[45] R.P. Fedkiw, T. Aslam, and S. Xu. The Ghost Fluid method for deflagration and detonation discontinuities. Journal of Computational Physics, 154:393-427, 1999.

[46] A. Ferrari, M. Dumbser, E.F. Toro, and A. Armanini. A new 3D parallel SPH scheme for free surface flows. Computers \& Fluids, 38:1203-1217, 2009.

[47] A. Ferrari, C.D. Munz, and B. Weigand. A High Order Sharp Interface Method with Local Timestepping for Compressible Multiphase Flows. Communications in Computational Physics, 9:205-230, 2011.

[48] J. Flaherty, R. Loy, M. Shephard, B. Szymanski, J. Teresco, and L. Ziantz. Adaptive local refinement with octree load-balancing for the parallel solution of three-dimensional conservation laws. J. Parallel Distrib. Comput., 47:139-152, 1997.

[49] J.M. Gallardo, C. Parés, and M.J. Castro. On a well-balanced highorder finite volume scheme for shallow water equations with topography and dry areas. Journal of Computational Physics, 227:574-601, 2007.

[50] G. Gassner, F. Lörcher, and C. D. Munz. A discontinuous Galerkin scheme based on a space-time expansion II. viscous flow equations in multi dimensions. Journal of Scientific Computing, 34:260-286, 2008.

[51] A. Harten, B. Engquist, S. Osher, and S. Chakravarthy. Uniformly high order essentially non-oscillatory schemes, III. Journal of Computational Physics, 71:231-303, 1987.

[52] C. Helzel, R.J. Leveque, and G. Warnecke. A modified fractional step method for the accurate approximation of detonation waves. SIAM Journal on Scientific Computing, 22:1489-1510, 2001.

[53] A. Hidalgo and M. Dumbser. ADER schemes for nonlinear systems of stiff advection-diffusion-reaction equations. Journal of Scientific Computing, 48:173-189, 2011.

[54] G.-S. Jiang and C.W. Shu. Efficient implementation of weighted ENO schemes. Journal of Computational Physics, pages 202-228, 1996. 
[55] A.K. Kapila, R. Menikoff, J.B. Bdzil, S.F. Son, and D.S. Stewart. Two-phase modelling of DDT in granular materials: reduced equations. Physics of Fluids, 13:3002-3024, 2001.

[56] R. Keppens, M. Nool, G. Tóth, and J.P Goedbloed. Adaptive Mesh Refinement for conservative systems: multi-dimensional efficiency evaluation . Computer Physics Communications, 153:317-339, 2003.

[57] A.M Khokhlov. Fully threaded tree algorithms for adaptive refinement fluid dynamics simulations. Journal of Computational Physics, 143(2):519 - 543, 1998.

[58] L. Krivodonova. An efficient local time-stepping scheme for solution of nonlinear conservation laws. Journal of Computational Physics, 229:8537-8551, 2010.

[59] A. Kurganov and G. Petrova. Central-upwind schemes on triangular grids for hyperbolic systems of conservation laws. Numerical Methods for Partial Differential Equations, 21(3):536-552, 2005.

[60] B.J. Lee, E.F. Toro, C.E. Castro, and N. Nikiforakis. Adaptive Osher-type scheme for the Euler equations with highly nonlinear equations of state. Journal of Computational Physics. in press. http://dx.doi.org/10.1016/j.jcp.2013.03.046.

[61] W. Liu, J. Cheng, and C.W. Shu. High order conservative Lagrangian schemes with Lax-Wendroff type time discretization for the compressible Euler equations. Journal of Computational Physics, 228:8872-8891, 2009 .

[62] R. Löhner. An adaptive finite element scheme for transient problems in CFD. Computer Methods in Applied Mechanics and Engineering, 61:323-338, April 1987.

[63] F. Lörcher, G. Gassner, and C. D. Munz. A discontinuous Galerkin scheme based on a space-time expansion. I. inviscid compressible flow in one space dimension. Journal of Scientific Computing, 32:175-199, 2007. 
[64] P.H. Maire. A high-order one-step sub-cell force-based discretization for cell-centered lagrangian hydrodynamics on polygonal grids. Computers and Fluids, 46(1):341-347, 2011.

[65] G. Dal Maso, P.G. LeFloch, and F. Murat. Definition and weak stability of nonconservative products. J. Math. Pures Appl., 74:483-548, 1995.

[66] O. Le Métayer, J. Massoni, and R. Saurel. Modelling evaporation fronts with reactive Riemann solvers. Journal of Computational Physics, 205:567-610, 2005.

[67] W. Mulder, S. Osher, and J.A. Sethian. Computing interface motion in compressible gas dynamics. Journal of Computational Physics, 100:209228, 1992.

[68] L.O. Müller, C. Parés, and E.F. Toro. Well-balanced high-order numerical schemes for one-dimensional blood flow in vessels with varying mechanical properties. Journal of Computational Physics, 242:53-85, 2013.

[69] A. Murrone and H. Guillard. A five equation reduced model for compressible two phase flow problems. Journal of Computational Physics, 202:664-698, 2005.

[70] D.A. Olivieri, M. Fairweather, and S.A.E.G. Falle. An adaptive mesh refinement method for solution of the transported PDF equation. International Journal for Numerical Methods in Engineering, 79:1536-1556, 2009.

[71] D.A. Olivieri, M. Fairweather, and S.A.E.G. Falle. Rans modelling of intermittent turbulent flows using adaptive mesh refinement methods. Journal of Turbulence, 11:1-18, 2010.

[72] S. Osher and J.A. Sethian. Fronts propagating with curvaturedependent speed: Algorithms based on Hamilton-Jacobi formulations. Journal of Computational Physics, 79:12-49, 1988.

[73] C. Parés. Numerical methods for nonconservative hyperbolic systems: a theoretical framework. SIAM Journal on Numerical Analysis, 44:300$321,2006$. 
[74] C. Parés and M.J. Castro. On the well-balance property of roe's method for nonconservative hyperbolic systems. applications to shallow-water systems. Mathematical Modelling and Numerical Analysis, 38:821-852, 2004 .

[75] E.B. Pitman and L. Le. A two-fluid model for avalanche and debris flows. Phil. Trans. R. Soc. A, 363:1573-1601, 2005.

[76] J.J. Quirk. A parallel adaptive grid algorithm for computational shock hydrodynamics. Applied Numerical Mathematics, 20(4):427 - 453, 1996.

[77] J.J. Quirk and S. Karni. On the dynamics of a shock-bubble interaction. Journal of Fluid Mechanics, 318:129-163, 1996.

[78] R. Saurel and R. Abgrall. A Multiphase Godunov Method for Compressible Multifluid and Multiphase Flows. Journal of Computational Physics, 150:425-467, 1999.

[79] R. Saurel, F. Petitpas, and R.A. Berry. Simple and efficient relaxation methods for interfaces separating compressible fluids, cavitating flows and shocks in multiphase mixtures. Journal of Computational Physics, 228:1678-1712, 2009.

[80] D.W. Schwendeman, C.W. Wahle, and A.K. Kapila. The Riemann problem and a high-resolution Godunov method for a model of compressible two-phase flow. Journal of Computational Physics, 212:490-526, 2006.

[81] A. Taube, M. Dumbser, C.D. Munz, and R. Schneider. A High Order Discontinuous Galerkin Method with Local Time Stepping for the Maxwell Equations. International Journal Of Numerical Modelling: Electronic Networks, Devices And Fields, 22:77-103, 2009.

[82] R. Teyssier. Cosmological hydrodynamics with adaptive mesh refinement. A new high resolution code called RAMSES. Astronomy $\& 3 \mathrm{As}$ trophysics, 385:337-364, April 2002.

[83] V.A. Titarev and E.F. Toro. ADER: Arbitrary high order Godunov approach. Journal of Scientific Computing, 17:609-618, 2002.

[84] V.A. Titarev and E.F. Toro. Finite-volume weno schemes for threedimensional conservation laws. Journal of Computational Physics, 201:238-260, 2004. 
[85] V.A. Titarev and E.F. Toro. ADER schemes for three-dimensional nonlinear hyperbolic systems. Journal of Computational Physics, 204:715736, 2005.

[86] V.A. Titarev and E.F. Toro. WENO schemes based on upwind and centred TVD fluxes. Computers and Fluids, 34:705-720, 2005.

[87] S.A. Tokareva and E.F. Toro. HLLC-type Riemann solver for the BaerNunziato equations of compressible two-phase flow. Journal of Computational Physics, 229(10):3573 - 3604, 2010.

[88] E.F. Toro. Riemann Solvers and Numerical Methods for Fluid Dynamics. Springer, third edition, 2009.

[89] E.F. Toro, R.C. Millington, and L.A.M Nejad. Towards very high order Godunov schemes. In E.F. Toro, editor, Godunov Methods. Theory and Applications, pages 905-938. Kluwer/Plenum Academic Publishers, 2001.

[90] E.F. Toro and V.A. Titarev. TVD fluxes for the high-order ADER schemes. Journal of Scientific Computing, 24:285-309, 2005.

[91] I. Toumi. A weak formulation of Roe's approximate Riemann solver. Journal of Computational Physics, 102:360-373, 1992.

[92] J. Utzmann, T. Schwartzkopff, M. Dumbser, and C.D. Munz. Heterogeneous Domain Decomposition for Computational Aeroacoustics. AIAA Journal, 44:2231-2250, 2006.

[93] A. Zein, M. Hantke, and G. Warnecke. Modeling phase transition for compressible two-phase flows applied to metastable liquids. Journal of Computational Physics, 229:2964-2998, 2010.

[94] Z.Q. Zhou, J. O. D. Kat, and B. Buchner. A nonlinear 3D approach to simulate green water dynamics on deck. 7th Int. Conf. Numer. Ship Hydrodyn., pages 1-4, 1999.

[95] U. Ziegler. The nirvana code: Parallel computational mhd with adaptive mesh refinement. Computer Physics Communications, 179(4):227 - 244, 2008. 